\title{
Light charged Higgs boson with dominant decay to a charm quark and a bottom quark and its search at LEP2 and future $e^{+} e^{-}$colliders
}

\author{
A. G. Akeroyd $\odot,{ }^{*}$ Stefano Moretti, ${ }^{\dagger}$ and Muyuan Song ${ }^{\ddagger}$ \\ School of Physics and Astronomy, University of Southampton, \\ Highfield, Southampton SO17 1BJ, United Kingdom
}

(Received 21 August 2019; accepted 27 January 2020; published 18 February 2020)

\begin{abstract}
The possibility of a light charged Higgs boson $H^{ \pm}$that decays predominantly to a charm quark and a bottom quark $(c b)$ and with a mass in the range $80 \mathrm{GeV} \leq M_{H^{ \pm}} \leq 90 \mathrm{GeV}$ is studied in the context of a three-Higgs doublet model (3HDM). Searches for this decay at the Large Hadron Collider do not have sensitivity to this mass region at present. It is shown that the searches for $H^{ \pm}$at LEP2 could be supplemented by either one or two $b$-tags, which would enable such large branching ratios for $H^{ \pm} \rightarrow c b$ to be probed in the above mass region. We comment on the possibility of this 3HDM scenario to explain a slight excess in the searches for $H^{ \pm}$at LEP2, which is best fit by $M_{H^{ \pm}}$of around $90 \mathrm{GeV}$, and discuss the prospects for detecting $H^{ \pm} \rightarrow c b$ decays at future $e^{+} e^{-}$colliders.
\end{abstract}

DOI: 10.1103/PhysRevD.101.035021

\section{INTRODUCTION}

The ATLAS and CMS [1,2] Collaborations at the CERN Large Hadron Collider (LHC) announced the discovery of a new particle (a spinless boson) with a mass of $125 \mathrm{GeV}$. The measurements of its properties (couplings, spin, etc.) are in excellent agreement with those of the Higgs boson of the Standard Model (SM), in which the Higgs boson originates from an $S U(2) \otimes U(1)$ scalar doublet.

It is possible that the $125 \mathrm{GeV}$ boson is the first scalar to be discovered from a nonminimal Higgs sector. An (singly) electrically charged Higgs boson $H^{ \pm}$would represent a distinctive signal of such a structure (see Ref. [3] for a recent phenomenological review) that could include additional doublets, singlets, triplets, or combinations thereof. There is considerable interest in Beyond the SM (BSM) scenarios with such a framework for implementing the Higgs mechanism of electroweak symmetry breaking. First, the SM is nonminimal in both its matter (with three fermionic generations) and gauge (with both strong and EW force mediators) sectors, and so there is no compelling reason to believe that the Higgs sector should be minimal. Second, in some BSM scenarios, an enlarged Higgs sector is required theoretically (e.g., supersymmetry) or provides

\footnotetext{
*a.g.akeroyd@soton.ac.uk

†.Moretti@soton.ac.uk

ms32g13@soton.ac.uk
}

Published by the American Physical Society under the terms of the Creative Commons Attribution 4.0 International license. Further distribution of this work must maintain attribution to the author(s) and the published article's title, journal citation, and DOI. Funded by SCOAP ${ }^{3}$. an explanation to problems that are not solved in the SM (e.g., necessity of nonzero neutrino masses, requirement of a dark matter candidate, sufficient EW baryogenesis, etc.).

The two-Higgs doublet model (2HDM) [4,5] has attracted the most attention among models with additional scalar doublets. Two (softly broken) discrete $Z_{2}$ symmetries are imposed in order to ensure that each fermion type couples to no more than one scalar doublet, leading to four distinct $2 \mathrm{HDMs}$ that differ in their Yukawa couplings. This framework, referred to as "natural flavor conservation" (NFC) [6], is invoked in order to avoid flavor changing neutral currents (FCNCs) that are mediated at tree-level by neutral scalars. More recently, three-Higgs doublet models (3HDMs) have received increased attention (see, e.g., Refs. [7,8] for minireviews), with NFC leading to five distinct 3HDMs.

Regarding the particle content of the $3 \mathrm{HDM}$, there are two physical charged Higgs bosons (hereafter denoted by $H^{ \pm}$and $H^{\prime \pm}$, with $M_{H^{ \pm}}<M_{H^{ \pm}}$). More parameters determine the phenomenology of the charged Higgs sector than in 2HDMs, and we make the assumption that all three Higgs doublets have a vacuum expectation value (VEV). In Refs. [9-13], the phenomenology of $H^{ \pm}$in 3HDMs has been studied (with decoupled $H^{ \pm}$) in terms of effective Yukawa couplings for the down-type quark, up-type quark, and charged lepton, which are expressed as a function of four independent parameters [11] in the framework of NFC. It has been shown [13] that an $H^{ \pm}$can be lighter than the top quark (with $H^{\prime \pm}$ heavier) while satisfying constraints from $B \rightarrow X_{s} \gamma$ (even for the Yukawa coupling combinations that would not permit this scenario in the 2HDM) due to the increased number of parameters in the $3 \mathrm{HDM}$ and the presence of two charged scalars. Moreover, it was shown in 
Refs. $[9,10,12,14]$ that the decay channel $H^{+} \rightarrow c \bar{b}$ can have a large branching ratio (BR) (up to $80 \%$ ) in a 3HDM. Although such a value for this BR is theoretically allowed in the flipped 2HDM for $M_{H^{ \pm}}<m_{t}-m_{b}$ [10], the constraint $M_{H^{ \pm}}>570 \mathrm{GeV}$ from $B \rightarrow X_{s} \gamma$ rules out this possibility [15-17]. Hence, a large $\operatorname{BR}\left(H^{+} \rightarrow c \bar{b}\right)$ is a distinctive signature of $3 \mathrm{HDMs}$.

The above scenario of a 3 HDM in which there is a light $H^{ \pm}$with a large BR to $c \bar{b}$ is the focus of this work. We consider the mass range $M_{H^{ \pm}} \sim M_{W^{ \pm}}$for which detection of $H^{ \pm}$is challenging if its BRs to hadrons are dominant. The LHC experimental collaborations have carried out searches at $\sqrt{s}=8 \mathrm{TeV}$ for $H^{ \pm} \rightarrow c b[18]$ and $H^{ \pm} \rightarrow$ hadrons $[19,20]$, assuming production via the mechanism $t \rightarrow H^{ \pm} b$, and the former search employs one more $b$-tag than the latter search. In Ref. [21], the parameter space in the flipped 3HDM that will be excluded (or provide a signal) at upcoming searches was displayed. At present, the LHC has not set limits in the region $80 \mathrm{GeV} \leq M_{H^{ \pm}} \leq 90 \mathrm{GeV}$ if $\mathrm{BR}\left(H^{+} \rightarrow c \bar{b}\right)$ or $\operatorname{BR}\left(H^{+} \rightarrow c \bar{s}\right)$ is dominant, although limits are set for the case of $H^{ \pm} \rightarrow \tau \nu$ being the leading decay channel. As discussed in [21], with the increased luminosity for the data taken at $\sqrt{s}=13 \mathrm{TeV}$ and with future data it is likely that the LHC will be able to set limits on $\operatorname{BR}\left(t \rightarrow H^{+} b\right) \times$ $\mathrm{BR}\left(H^{+} \rightarrow c \bar{b}\right)$ in part (if not all) of the region $80 \mathrm{GeV} \leq$ $M_{H^{ \pm}} \leq 90 \mathrm{GeV}$ (and possibly in the case of the $H^{+} \rightarrow c \bar{s}$ channel as well). However, the production mechanism relies on the Yukawa couplings and thus such an $H^{ \pm}$could escape detection at the LHC if these couplings are small. Consequently, it is of interest to study in more detail the CERN LEP2 searches for a hadronically decaying $H^{ \pm}$, for which the main production mode of $e^{+} e^{-} \rightarrow H^{+} H^{-}$ depends only on gauge couplings and $M_{H^{ \pm}}$.

We will show that data taken at LEP2 when supplemented by $b$-tagging could discover or exclude a light $H^{ \pm}$ state decaying to $c \bar{b}$ pairs more efficiently than LHC searches in the region $80 \mathrm{GeV} \leq M_{H^{ \pm}} \leq 90 \mathrm{GeV}$. Before the LEP2 era, this possibility was pointed out for models with more than two Higgs doublets in Refs. [10,14], although the brief quantitative study in [14] (that was based on a simulation in [22]) concluded that sensitivity would not be reached in the region $80 \mathrm{GeV} \leq M_{H^{ \pm}} \leq 90 \mathrm{GeV}$. Such a $b$-tag was never implemented in LEP2 searches for $H^{ \pm}$states. We revisit it here in the context of the flipped 3HDM and show that by using $b$-quark tagging and light-quark rejection efficiencies from the LEP2 searches, one can substantially improve the sensitivity to $H^{ \pm} \rightarrow c b$ compared to that for $H^{ \pm} \rightarrow$ hadrons and probe the region $80 \mathrm{GeV} \leq M_{H^{ \pm}} \leq 90 \mathrm{GeV}$. Attention is also given to the detection prospects for $H^{ \pm} \rightarrow c b$ at future $e^{+} e^{-}$ colliders operating at $\sqrt{s}=240 \mathrm{GeV}$.

The plan of this paper is as follows. In Sec. II, the 3HDM is introduced. In Sec. III, the LEP2 search for $H^{ \pm}$with the addition of $b$-tagging is described, with numerical results and conclusions in Secs. IV and V, respectively.

\section{THE 3HDM WITH NFC}

In this section, we give a brief introduction to the interactions of the lightest $H^{ \pm}$in the 3HDM that are relevant to our analysis. We will only consider $M_{H^{ \pm}}<m_{t}$, and we assume that the only channels that have nonzero BRs are the decays to fermions (i.e., decays of the type $H^{ \pm} \rightarrow W^{ \pm}$plus a neutral Higgs boson are forbidden by setting the masses of all the neutral Higgs bosons to be above that of the charged Higgs). For a more detailed introduction, the reader is referred to $[11,13]$.

Any extension of the SM Higgs sector is primarily constrained by two experimental facts. First, the measurement of $\rho=m_{W}^{2} /\left(m_{Z}^{2} \cos ^{2} \theta_{W}\right)$ is close to 1 [4], where $m_{W}$, $m_{Z}$, and $\theta_{W}$ are the $W, Z$ masses and weak mixing angle, respectively. Second, tree-level FCNCs that are mediated by the additional neutral scalars must be suppressed (or absent). In order for the 3HDM to comply with both of the above restrictions, one requires (i) no very large mass splittings between the neutral and charged scalars in order to respect $\rho$ parameter bounds and (ii) to implement NFC [6] in order to eliminate tree-level FCNCs. The most general $S U(2) \otimes$ $U(1)_{Y}$ invariant scalar potential $(V)$ is given by [11]

$$
\begin{aligned}
V= & m_{11}^{2} \Phi_{1}^{\dagger} \Phi_{1}+m_{22}^{2} \Phi_{2}^{\dagger} \Phi_{2}+m_{33}^{2} \Phi_{3}^{\dagger} \Phi_{3}-\left[m_{12}^{2} \Phi_{1}^{\dagger} \Phi_{2}+m_{13}^{2} \Phi_{1}^{\dagger} \Phi_{3}+m_{23}^{2} \Phi_{2}^{\dagger} \Phi_{3}+\text { H.c. }\right]+\frac{1}{2} \lambda_{1}\left(\Phi_{1}^{\dagger} \Phi_{1}\right)^{2}+\frac{1}{2} \lambda_{2}\left(\Phi_{2}^{\dagger} \Phi_{2}\right)^{2} \\
& +\frac{1}{2} \lambda_{3}\left(\Phi_{3}^{\dagger} \Phi_{3}\right)^{2}+\lambda_{12}\left(\Phi_{1}^{\dagger} \Phi_{1}\right)\left(\Phi_{2}^{\dagger} \Phi_{2}\right)+\lambda_{13}\left(\Phi_{1}^{\dagger} \Phi_{1}\right)\left(\Phi_{3}^{\dagger} \Phi_{3}\right)+\lambda_{23}\left(\Phi_{2}^{\dagger} \Phi_{2}\right)\left(\Phi_{3}^{\dagger} \Phi_{3}\right)+\lambda_{12}^{\prime}\left(\Phi_{1}^{\dagger} \Phi_{2}\right)\left(\Phi_{2}^{\dagger} \Phi_{1}\right) \\
& +\lambda_{13}^{\prime}\left(\Phi_{1}^{\dagger} \Phi_{3}\right)\left(\Phi_{3}^{\dagger} \Phi_{1}\right)+\lambda_{23}^{\prime}\left(\Phi_{2}^{\dagger} \Phi_{3}\right)\left(\Phi_{3}^{\dagger} \Phi_{2}\right)+\frac{1}{2}\left[\lambda_{12}^{\prime \prime}\left(\Phi_{1}^{\dagger} \Phi_{2}\right)^{2}+\lambda_{13}^{\prime \prime}\left(\Phi_{1}^{\dagger} \Phi_{3}\right)^{2}+\lambda_{23}^{\prime \prime}\left(\Phi_{2}^{\dagger} \Phi_{3}\right)^{2}+\text { H.c. }\right] .
\end{aligned}
$$

The scalar doublets $\Phi_{f}$ (with $f=1,2,3$ ) are defined as follows:

$$
\Phi_{f}=\left(\begin{array}{c}
\phi_{f}^{+} \\
\left(v_{f}+\phi_{f}^{0, r}+i \phi_{f}^{0, i}\right) / \sqrt{2}
\end{array}\right) .
$$

Here $\phi_{f}^{0}$ and $\phi_{f}^{+}$denote neutral and (positively) charged scalar fields, respectively, and the VEVs $v_{f}$ are nonzero for each doublet. There are two physical charged scalars $\left(H^{ \pm}\right.$ and $H^{ \pm \pm}$), two neutral charge parity $(C P)$-odd scalars, and three $C P$-even scalars. In the simplified case of all the 
parameters being real, there are 18 free parameters in the scalar potential. However, two of these parameters are determined by the mass of the $W$ boson and the mass of the $125 \mathrm{GeV}$ neutral Higgs boson, leaving (at least) 16 free parameters.

In our numerical analysis for the lightest charged scalar $H^{ \pm}$, we are only concerned with five of these 16 (or more) free parameters in the scalar potential of the $3 \mathrm{HDM}$, as explained later in this section. There are theoretical constraints on these 16 parameters from requiring the stability of the vacuum, the absence of charge breaking minima, and compliance with unitarity of scattering processes, etc. These constraints are wellknown in the 2HDM (e.g., see [23]) and have been discussed for the scalar potential of the 3HDM in [24,25]. In this work, we do not impose these constraints because they would only exclude certain regions of the parameter space of 16 variables. As will be discussed later in this section, the phenomenology of $H^{ \pm}$depends on only five parameters; four of these arise from a mixing matrix (and also determine the phenomenology of $H^{\prime \pm}$ ) and the fifth is $M_{H^{ \pm}}$. We take these parameters to be theoretically unconstrained, but they are constrained experimentally. It is assumed that the freedom in the remaining 11 parameters can be used to respect the above theoretical constraints while allowing the five parameters that determine the phenomenology of $H^{ \pm}$to be varied in their full theoretical range. Our justification for this approach is that the analogous constraints on the scalar potential in $2 \mathrm{HDMs}$ do not restrict the allowed ranges of the two free parameters in the charged Higgs sector $\left(M_{H^{ \pm}}\right.$and $\left.\tan \beta\right)$ because of the freedom in the remaining four parameters (for the case of a $2 \mathrm{HDM}$ scalar potential with only soft breaking terms of a $Z_{2}$ symmetry). It is experimental data from processes involving $H^{ \pm}$that constrain the ranges of the parameters of the charged Higgs sector in a 2HDM, and we carry this conclusion across to the charged Higgs sector of the 3HDM.

Experimental constraints on the neutral scalars of the 3HDM from direct searches (including the discovery of the $125 \mathrm{GeV}$ Higgs boson) will only constrain a combination of parameters (of 16 or more) that are relevant for the neutral Higgs sector. Compliance with electroweak precision observables can be obtained by not having large mass splittings among the scalars, although we expect that the allowed mass splittings in a 3HDM would be larger than those in a $2 \mathrm{HDM}$ due to the increased number of physical scalars and free parameters. Hence, we expect that a sizeable splitting between $H^{ \pm}$and $H^{\prime \pm}$ (which we use later when applying a bound from $b \rightarrow s \gamma$ ) would be acceptable in a $3 \mathrm{HDM}$.

The part of the Yukawa Lagrangian containing the lightest charged Higgs boson interactions with the fermions can be written as follows:

$$
\begin{aligned}
\mathcal{L}_{H^{ \pm}}= & -H^{+}\left\{\frac{\sqrt{2} V_{u d}}{v_{\mathrm{SM}}} \bar{u}\left(m_{d} X P_{R}+m_{u} Y P_{L}\right) d\right. \\
& \left.+\frac{\sqrt{2} m_{\ell}}{v_{\mathrm{SM}}} Z \bar{\nu}_{L} \ell_{R}\right\}+ \text { H.c. }
\end{aligned}
$$

Here $u(d)$ is denotes up(down)-type quarks and $\ell$ represents charged leptons, $P_{L(R)}$ is the left(right)-handed projector, $V_{u d}$ is the relevant Cabibbo-Kobayashi-Maskawa (CKM) matrix element, and $v_{\mathrm{SM}}$ is the VEV of the Higgs doublet in the SM. In the 3HDM, the couplings $X, Y$, and $Z$ are functions of the four parameters (see below) of a unitary matrix $U$ that connects the charged scalar interaction eigenstates to the physical mass eigenstates as follows:

$$
\left(\begin{array}{c}
G^{+} \\
H^{+} \\
H^{\prime+}
\end{array}\right)=U\left(\begin{array}{c}
\phi_{1}^{+} \\
\phi_{2}^{+} \\
\phi_{3}^{+}
\end{array}\right)
$$

Here $H^{+}, H^{\prime+}$ are physical charged scalars, whereas $G^{+}$is a charged Goldstone boson that will become the longitudinal component of the $W^{ \pm}$gauge boson after electroweak symmetry breaking. The matrix $U$ is a $3 \times 3$ unitary matrix and can be parametrized as a function of four parameters, $\tan \beta, \tan \gamma, \theta$, and $\delta$. The first two parameters are defined via

$$
\tan \beta=v_{2} / v_{1}, \quad \tan \gamma=\sqrt{v_{1}^{2}+v_{2}^{2}} / v_{3},
$$

where $v_{1}, v_{2}$, and $v_{3}$ are the VEVs of each Higgs doublet. The parameter $\theta$ is a mixing angle between the two massive charged scalars and $\delta$ is a $C P$-violating phase. The explicit form of $U$ is as follows [11]:

$U=\left(\begin{array}{ccc}s_{\gamma} c_{\beta} & s_{\gamma} s_{\beta} & c_{\gamma} \\ -c_{\theta} s_{\beta} e^{-i \delta}-s_{\theta} c_{\gamma} c_{\beta} & c_{\theta} c_{\beta} e^{-i \delta}-s_{\theta} c_{\gamma} s_{\beta} & s_{\theta} s_{\gamma} \\ s_{\theta} s_{\beta} e^{-i \delta}-c_{\theta} c_{\gamma} c_{\beta} & -s_{\theta} c_{\beta} e^{-i \delta}-c_{\theta} c_{\gamma} s_{\beta} & c_{\theta} s_{\gamma}\end{array}\right)$,

where $s(c)$ are represents the sine(cosine) of the respective angle.

The interactions between the lightest charged Higgs state of the 3HDM, $H^{ \pm}$, and the SM fermions are obtained via the $U$ matrix as [9]

$$
X=\frac{U_{d 2}^{\dagger}}{U_{d 1}^{\dagger}}, \quad Y=-\frac{U_{u 2}^{\dagger}}{U_{u 1}^{\dagger}}, \quad Z=\frac{U_{\ell 2}^{\dagger}}{U_{\ell 1}^{\dagger}},
$$

where the values of $d, u$, and $\ell$ in these matrix elements are given in Table I and depend upon which of the five possible distinct $3 \mathrm{HDMs}$ are under consideration. Taking $d=1$, $u=2$, and $\ell=3$ means that the down-type quarks receive their mass from $v_{1}$, the up-type quarks from $v_{2}$, and the 
TABLE I. The five versions of the 3HDM with NFC and the corresponding $u, d$, and $\ell$ values. Taking $u=i$ means that the uptype quarks receive their mass from $v_{i}$ and likewise for $d$ (downtype quarks) and $\ell$ (charged leptons).

\begin{tabular}{llll}
\hline \hline & $u$ & $d$ & $\ell$ \\
\hline 3HDM (type I) & 2 & 2 & 2 \\
3HDM (type II) & 2 & 1 & 1 \\
3HDM (lepton-specific) & 2 & 2 & 1 \\
3HDM (flipped) & 2 & 1 & 2 \\
3HDM (democratic) & 2 & 1 & 3 \\
\hline \hline
\end{tabular}

charged leptons from $v_{3}$. This choice is called the "democratic 3HDM," while the other possible choices of $d, u$, and $\ell$ in a 3HDM are given the same names as the four standard types of 2HDM [5].

The experimental constraints on $X, Y$, and $Z[26,27]$ have been summarized in Ref. [21], to which we refer the reader. The parameter space of the $3 \mathrm{HDM}$ that is relevant to this work is compliant with all such limits, the most important of which being $-1.1<\operatorname{Re}\left(X Y^{*}\right)<0.7$ for $M_{H^{ \pm}}<100 \mathrm{GeV}$. This is an approximate constraint that is derived from $b \rightarrow s \gamma$ in the context of the aligned 2HDM [26,27], which has one $H^{ \pm}$. Applying this bound to the lightest $H^{ \pm}$of a $3 \mathrm{HDM}$ requires that the contribution of the heavier $H^{ \pm}$in a $3 \mathrm{HDM}$ is suppressed, which can be arranged by taking $H^{\prime \pm}$ to have a considerably larger mass.

In a $3 \mathrm{HDM}$, the expressions for the partial widths of the decay of $H^{ \pm}$to fermions are as follows:

$$
\begin{gathered}
\Gamma\left(H^{ \pm} \rightarrow \ell^{ \pm} \nu\right)=\frac{G_{F} M_{H^{ \pm}} m_{\ell}^{2}|Z|^{2}}{4 \pi \sqrt{2}}, \\
\Gamma\left(H^{ \pm} \rightarrow u d\right)=\frac{3 G_{F} V_{u d} M_{H^{ \pm}}\left(m_{d}^{2}|X|^{2}+m_{u}^{2}|Y|^{2}\right)}{4 \pi \sqrt{2}} .
\end{gathered}
$$

In the expression for $\Gamma\left(H^{ \pm} \rightarrow u d\right)$, the running quark masses should be evaluated at the scale of $m_{H^{ \pm}}$, and there are QCD vertex corrections which multiply the partial widths by $\left(1+17 \alpha_{s} /(3 \pi)\right)$. The first study of the fermionic BRs of $H^{ \pm}$as a function of $|X|,|Y|$, and $|Z|$ was given in [10], with further studies in [12]. In $[13,21]$, these BRs were studied as a function of $\tan \beta, \tan \gamma, \theta$, and $\delta$, an approach which allows the BRs in the five versions of the 3HDM to be compared. For $|X| \gg|Y|,|Z|$, the decay channel $\operatorname{BR}\left(H^{ \pm} \rightarrow c b\right)$ dominates (which was first mentioned in [9]) and reaches a maximum of $\sim 80 \%$. It was shown in $[13,21]$ that such large values of $\operatorname{BR}\left(H^{ \pm} \rightarrow c b\right)$ are only possible in the flipped and democratic 3HDMs, with $\operatorname{BR}\left(H^{ \pm} \rightarrow c b\right)$ having a maximum value of around $1 \%$ in the other 3HDMs. In 2HDMs with NFC, the only model which contains a parameter space for a large $\mathrm{BR}\left(H^{ \pm} \rightarrow c b\right)$ with $M_{H^{ \pm}}<m_{t}$ is the flipped model (a possibility that was mentioned in $[9,10]$ and studied in more detail in [28]).
TABLE II. Searches for $H^{ \pm}$at the LHC, using $p p \rightarrow t \bar{t}$ and $t \rightarrow H^{ \pm} b$. The given integrated luminosities are approximate. The search in [31] used $2 \mathrm{fb}^{-1}$.

\begin{tabular}{lcc}
\hline \hline & ATLAS & CMS \\
\hline $7 \mathrm{TeV}\left(5 \mathrm{fb}^{-1}\right)$ & $c s[19], \tau \nu[32,33]$ & $\tau \nu[31]$ \\
$8 \mathrm{TeV}\left(20 \mathrm{fb}^{-1}\right)$ & $\tau \nu[34]$ & $c s[20], c b[18], \tau \nu[35]$ \\
$13 \mathrm{TeV}\left(36 \mathrm{fb}^{-1}\right)$ & $\tau \nu[36]$ & $\tau \nu[37]$ \\
\hline \hline
\end{tabular}

However, for this particular choice of 2HDM, the $b \rightarrow s \gamma$ constraint would require $M_{H^{ \pm}}>570 \mathrm{GeV}$ [15-17] for which $H^{ \pm} \rightarrow t b$ would dominate.

In this paper, we will focus on the case of $m_{H^{ \pm}}<m_{t}$, a scenario in which production at the LHC via $t \rightarrow H^{ \pm} b$ would be possible. Searches for three decays channels of $H^{ \pm}$ have been carried out (see Table II). The searches for $H^{ \pm} \rightarrow$ $\tau \nu$ constrain the product $\mathrm{BR}\left(t \rightarrow H^{ \pm} b\right) \times \mathrm{BR}\left(H^{ \pm} \rightarrow \tau \nu\right)$ in the region $80 \mathrm{GeV}<M_{H^{ \pm}}<160 \mathrm{GeV}$, with the upper limit ranging from $<0.36 \%$ for $M_{H^{ \pm}}=80 \mathrm{GeV}$ to $<$ $0.08 \%$ for $M_{H^{ \pm}}=160 \mathrm{GeV}$. The searches for $H^{ \pm} \rightarrow c s$ constrain the product $\mathrm{BR}\left(t \rightarrow H^{ \pm} b\right) \times \mathrm{BR}\left(H^{ \pm} \rightarrow c s\right)$ in the region $90 \mathrm{GeV}<M_{H^{ \pm}}<160 \mathrm{GeV}$, with the upper limit ranging from $<5 \%$ for $M_{H^{ \pm}}=90 \mathrm{GeV}$ to $<2 \%$ for $M_{H^{ \pm}}=160 \mathrm{GeV}$. Note that this search would be sensitive to any quark decay (except $t$ ) of $H^{ \pm}$. The search for $H^{ \pm} \rightarrow c b$ (which employs one more $b$-tag than the search for $\left.H^{ \pm} \rightarrow c s\right)$ constrains the product $\operatorname{BR}\left(t \rightarrow H^{ \pm} b\right) \times$ $\operatorname{BR}\left(H^{ \pm} \rightarrow c b\right)$, with the upper limit ranging from $<1.4 \%$ for $M_{H^{ \pm}}=90 \mathrm{GeV}$ to $<0.5 \%$ for $M_{H^{ \pm}}=150 \mathrm{GeV}$. The searches for $H^{ \pm} \rightarrow c s$ and $H^{ \pm} \rightarrow c b$ do not set limits on the region $80 \mathrm{GeV}<M_{H^{ \pm}}<90 \mathrm{GeV}$, although this might be possible (especially for $H^{ \pm} \rightarrow c b$ ) with larger integrated luminosities. Earlier searches for the decay $t \rightarrow H^{ \pm} b$ were carried out at the Fermilab Tevatron in $[29,30]$.

At LEP2, the production process $\sigma\left(e^{+} e^{-} \rightarrow \gamma^{*}, Z^{*} \rightarrow\right.$ $\left.H^{+} H^{-}\right)$was used. This cross section [38] is a function of well-measured SM parameters $\left(\alpha, \sin \theta_{W}, M_{Z}, \Gamma_{Z}\right)$, the center-of-mass energy $\sqrt{s}$, and $M_{H^{ \pm}}$(which is the only unknown parameter). Searches were carried out at all four experiments [39-42] at energies in the range $\sqrt{s}=$ $183 \mathrm{GeV}$ to $\sqrt{s}=209 \mathrm{GeV}$, each with an integrated luminosity of roughly $0.6 \mathrm{fb}^{-1}$. The LEP working group [43] combined these individual searches, resulting in a cumulative integrated luminosity of $2.6 \mathrm{fb}^{-1}$. Dedicated searches for the decay mode $H^{ \pm} \rightarrow A^{0} W^{*}$ were also carried out in $[39,42]$, but in this work we are assuming that this channel is absent or very suppressed. From the combination of the searches for fermionic decays, and with the assumption that $\mathrm{BR}\left(H^{ \pm} \rightarrow \tau \nu\right)+\mathrm{BR}\left(H^{ \pm} \rightarrow c s\right)=1$, the excluded region at $95 \%$ confidence level in the plane $\left[M_{H^{ \pm}}, \operatorname{BR}\left(H^{ \pm} \rightarrow \tau \nu\right)\right]$ is obtained in [43]. For $M_{H^{ \pm}}<$ $80 \mathrm{GeV}$, the whole range $0 \leq \mathrm{BR}\left(H^{ \pm} \rightarrow \tau \nu\right) \leq 100 \%$ is excluded. For $80 \mathrm{GeV} \leq M_{H^{ \pm}}<90 \mathrm{GeV}$, most of the 
region is not excluded for $\mathrm{BR}\left(H^{ \pm} \rightarrow \tau \nu\right)<80 \%$ (i.e., for $\left.\mathrm{BR}\left(H^{ \pm} \rightarrow c s\right)>20 \%\right)$. We will focus on this region of $80 \mathrm{GeV} \leq M_{H^{ \pm}}<90 \mathrm{GeV}$ and the case of a large hadronic BR for $H^{ \pm}$, which is not being probed by the LHC at present.

As mentioned earlier, the LHC searches for $t \rightarrow H^{ \pm} b$ with $H^{ \pm} \rightarrow \tau \nu$ are sensitive to the region $80 \mathrm{GeV}<M_{H^{ \pm}}<$ $90 \mathrm{GeV}$. However, for a given value of $M_{H^{ \pm}}$, it is the product $\operatorname{BR}\left(t \rightarrow H^{ \pm} b\right) \times \mathrm{BR}\left(H^{ \pm} \rightarrow \tau \nu\right)$ that is being constrained at the LHC, while at LEP2 it is $\operatorname{BR}\left(H^{ \pm} \rightarrow \tau \nu\right)$ alone that is being constrained for a given value of $M_{H^{ \pm}}$. A choice with $M_{H^{ \pm}}=85 \mathrm{GeV}$ and $\operatorname{BR}\left(H^{ \pm} \rightarrow \tau \nu\right)=0.5$ is not ruled out at LEP. At the $\mathrm{LHC}, \operatorname{BR}\left(t \rightarrow H^{ \pm} b\right) \times$ $\operatorname{BR}\left(H^{ \pm} \rightarrow \tau \nu\right)>0.36 \%$ is ruled out for $M_{H^{ \pm}}=85 \mathrm{GeV}$. Hence, if $\operatorname{BR}\left(t \rightarrow H^{ \pm} b\right)<0.72 \%$, then the above choice with $M_{H^{ \pm}}=85 \mathrm{GeV}$ and $\mathrm{BR}\left(H^{ \pm} \rightarrow \tau \nu\right)=0.5$ is also not ruled out at the LHC. Such values of $\operatorname{BR}\left(t \rightarrow H^{ \pm} b\right)$ can be arranged with suitably small values of $|X|$ and $|Y|$, which determine $\operatorname{BR}\left(t \rightarrow H^{ \pm} b\right)$. In contrast, it is the relative values of $|X|,|Y|$, and $|Z|$ that determine the fermionic BRs of $H^{ \pm}$(assuming that additional non-fermionic decay channels are not open).

\section{SEARCH FOR $H^{ \pm}$AT LEP2}

At LEP2, it was assumed that the dominant decay channels were $H^{ \pm} \rightarrow c s$ and $H^{ \pm} \rightarrow \tau \nu$, which leads to the following three signatures from $\mathrm{H}^{+} \mathrm{H}^{-}$production: $\operatorname{cscs}, \operatorname{cs\tau } \nu, \tau \nu \tau \nu$. The decay of $H^{ \pm} \rightarrow c b$ was not explicitly searched for at LEP2 [39-42]. It is the searches in the hadronic channels cscs and $\operatorname{cs\tau } \nu$ that are relevant for the decay $H^{ \pm} \rightarrow c b$, and these are discussed in more detail below.

(i) Four-jet channel: This signature arises when $\mathrm{H}^{+}$and $H^{-}$both decay into quarks, giving four quarks that will usually be detected as four jets. For $H^{ \pm}$in the kinematical range of LEP2 (i.e., $M_{H^{ \pm}}<\sqrt{s} / 2 \approx$ $100 \mathrm{GeV}$ ), there are six possible hadronic decay channels of $H^{ \pm}$. Decays involving the $t$ quark (e.g., $H^{ \pm} \rightarrow t^{*} b$ ) are extremely suppressed due to the $t$ quark being very off shell and can be neglected. In the LEP searches, it was assumed that $H^{ \pm} \rightarrow c s$ is the dominant hadronic decay mode, which is true in most 2HDMs, and the experimental limits on $\mathrm{BR}\left(H^{ \pm} \rightarrow\right.$ hadrons $)$ were interpreted as limits on $\operatorname{BR}\left(H^{ \pm} \rightarrow c s\right)$. However, the four-jet search as carried out by three of the LEP Collaborations (OPAL [39], ALEPH [40], L3 [41]) was sensitive to any of the allowed six decay channels into quarks. In contrast, the search by the DELPHI Collaboration [42] was optimized for $H^{ \pm} \rightarrow c s$ decays by using $c$-tagging to discriminate against lighter quarks and $b$ quarks, as well as requiring an identified kaon. This search strategy would probably be less sensitive to the decay $H^{ \pm} \rightarrow c b$ than the searches by the other three collaborations, because $H^{ \pm} \rightarrow c b$ events would not look exactly like $H^{ \pm} \rightarrow c s$ events.

(ii) Two-jet $+\tau \nu$ channel: This signature arises when one $H^{ \pm}$decays into quarks and the other $H^{ \pm}$decays into a $\tau$ lepton and a neutrino. Again, it was assumed that $H^{ \pm} \rightarrow c s$ is the dominant hadronic decay mode, and the DELPHI Collaboration alone used $c$-tagging.

In this work, we quantify the effect of applying one (or more) $b$-tags to both of the above search strategies in order to increase the sensitivity to the decay $H^{ \pm} \rightarrow c b$, which can have a large BR in the flipped and democratic 3HDMs. In the four-jet channel, the separate cases of exactly one tagged $b$-jet and exactly two tagged $b$-jets will be considered. In the two-jet $+\tau \nu$ channel, the case of exactly one tagged $b$-jet will be considered. A $b$-tag requirement usually involves a cut on the impact parameter of a jet [44]. Due to the longer lifetime of the $b$ quark, a jet that has originated from a $b$ quark will (on average) have a larger impact parameter than a jet that originated from a lighter quark. Additional discriminating variables are sometimes used in the full $b$-tag requirement. The three dominant decay channels of $H^{ \pm}$in the 3HDMs that we study are $\mathrm{BR}\left(H^{ \pm} \rightarrow c b\right), \mathrm{BR}\left(H^{ \pm} \rightarrow c s\right)$, and $\mathrm{BR}\left(H^{ \pm} \rightarrow \tau \nu\right)$. These will be denoted below by $B R_{c b}, B R_{c s}$, and $B R_{\tau \nu}$, respectively.

\section{A. Signal for $\boldsymbol{H}^{ \pm} \rightarrow \boldsymbol{c b}$ with $\boldsymbol{b}$-tags at LEP2}

The number of expected $e^{+} e^{-} \rightarrow H^{+} H^{-}$events (with no $b$-tag requirement) in the LEP2 searches in the four-jet and two-jet $+\tau \nu$ channels are denoted by $S_{4 \text { jnobtag }}$ and $S_{2 j \tau \text { nobtag }}$, respectively, and are given as follows:

(i) $S_{4 \text { jnobtag }}=\sigma \times \mathcal{L} \times \epsilon_{4 \text { jnobtag }} \times\left(B R_{c b}+B R_{c s}\right)^{2}$.

Note that $B R_{c b}$ and $B R_{c s}$ are summed, because the search strategy does not apply a $b$-tag.

(ii) $S_{2 j \text { nnobtag }}=\sigma \times \mathcal{L} \times \epsilon_{2 j \text { nnobtag }} \times 2\left(B R_{c b}+B R_{c s}\right) B R_{\tau \nu}$. Note that $B R_{c b}$ and $B R_{c s}$ are summed (as above), and the factor of 2 accounts for the separate contributions from $c \bar{s} \tau^{-} \bar{\nu}$ and $\bar{c} s \tau^{+} \nu$.

Here $\sigma$ is the cross section for pair production of $\mathrm{H}^{+} \mathrm{H}^{-}$ at a particular center-of-mass energy $\sqrt{s}$ (which we generate using the formulae for $\sigma\left(e^{+} e^{-} \rightarrow H^{+} H^{-}\right)$given in [38]), and $\mathcal{L}$ is integrated luminosity at that energy. The searches for $\mathrm{H}^{+} \mathrm{H}^{-}$at LEP2 were carried out using data taken at eight different values of $\sqrt{s}$, each with a unique value of integrated luminosity $\mathcal{L}$. Hence, the product $\sigma \mathcal{L}$ is actually a sum $\sum_{i=1}^{8} \sigma_{i} \mathcal{L}_{i}$ where each $i$ denotes a specific value of $\sqrt{s}$. Around $100 \mathrm{H}^{+} \mathrm{H}^{-}$events are produced (before cuts) for $M_{H^{ \pm}}=80 \mathrm{GeV}$ and $\sqrt{s}=200 \mathrm{GeV}$. The parameters $\epsilon_{4 \text { jnobtag }}$ and $\epsilon_{2 j \text { nnobtag }}$ are the selection efficiencies for the cuts as used in the LEP searches for the four-jet signature and the two-jet $+\tau \nu$ signature, respectively. For the magnitude of these efficiencies, we will use $\epsilon_{4 \text { jnobtag }}=$ 0.64 and $\epsilon_{2 j \text { nnobtag }}=0.5$. These values are similar to those 
that were obtained by the LEP2 Collaborations (with slight numerical differences among the four experiments). We now discuss in turn three proposed search strategies for the decay $H^{ \pm} \rightarrow c b$ that make use of $b$-tagging.

\section{Signal in four-jet channel with exactly two b-tagged jets}

A maximum of two $b$ quarks can be produced when both charged scalars decay via $H^{ \pm} \rightarrow c b$. However, lighter quarks $(u, d, s, c)$ can fake $b$ quarks, and so up to four jets could be recorded as $b$-jets by a detector. In the numerical analysis for LEP2, the $b$-tag efficiency $\left(\epsilon_{b}\right)$ is taken to be $\epsilon_{b}=0.7$, while the fake $b$-tag efficiencies for charm quarks $\left(\epsilon_{c}\right)$ and $u, d, s$ quarks $\left(\epsilon_{j}\right)$ are $\epsilon_{c}=0.06$ and $\epsilon_{j}=0.01$, respectively. These numbers are roughly similar (although slightly optimistic for $\epsilon_{b}$ ) to those in the OPAL measurement of $R_{b}$ in [45] for $\sqrt{s}=183 \mathrm{GeV}$ to $209 \mathrm{GeV}$. Due to $\epsilon_{c}$ and $\epsilon_{j}$ being small, we will not consider the signatures of three or four tagged $b$-jets, in which one or two non- $b$ quarks have been mistagged as $b$ quarks. We first consider the channel in which exactly two of the four jets are tagged as $b$-jets. The number of such events is denoted by $S_{4 j 2 b t a g}$ and is given by the following expression:

$$
\begin{aligned}
S_{4 j 2 b t a g}= & \sigma \times \mathcal{L} \times \epsilon_{4 j \text { nobtag }} \times\left(B R_{c b} B R_{c b} \epsilon_{4 j 2 \mathrm{btag}}^{c b c b}\right. \\
& \left.+2 B R_{c b} B R_{c s} \epsilon_{4 j 2 \mathrm{btag}}^{c b c s}+B R_{c s} B R_{c s} \epsilon_{4 j 2 \mathrm{btag}}^{c s c s}\right) .
\end{aligned}
$$

The factor of 2 accounts for the $c \bar{b} \bar{c} s$ and $\bar{c} b c \bar{s}$ signatures. This expression for $S_{4 j 2 \mathrm{btag}}$ is obtained from the expression for $S_{4 \text { jnobtag, }}$, with the effect of the $b$-tagging requirement contained in the parameters $\epsilon_{4 j 2 b t a g}^{c b c b}, \epsilon_{4 j 2 \text { btag }}^{c b c s}$, and $\epsilon_{4 j 2 b t a g}^{c s c s}$ that are given explicitly as follows:

$$
\begin{aligned}
\epsilon_{4 j 2 \mathrm{btag}}^{c b c b} & =\epsilon_{b}^{2}\left(1-\epsilon_{c}\right)^{2}+4 \epsilon_{b} \epsilon_{c}\left(1-\epsilon_{b}\right)\left(1-\epsilon_{c}\right)+\epsilon_{c}^{2}\left(1-\epsilon_{b}\right)^{2}, \\
\epsilon_{4 j 2 \mathrm{btag}}^{c b c s}= & 2 \epsilon_{b} \epsilon_{c}\left(1-\epsilon_{c}\right)\left(1-\epsilon_{j}\right)+\epsilon_{b} \epsilon_{j}\left(1-\epsilon_{c}\right)^{2} \\
& +2 \epsilon_{c} \epsilon_{j}\left(1-\epsilon_{b}\right)\left(1-\epsilon_{c}\right)+\epsilon_{c}^{2}\left(1-\epsilon_{b}\right)\left(1-\epsilon_{j}\right), \\
\epsilon_{4 j \mathrm{bbtag}}^{c s c c s} & =4 \epsilon_{c} \epsilon_{j}\left(1-\epsilon_{c}\right)\left(1-\epsilon_{j}\right)+\epsilon_{c}^{2}\left(1-\epsilon_{j}\right)^{2}+\epsilon_{j}^{2}\left(1-\epsilon_{c}\right)^{2} .
\end{aligned}
$$

Inserting the above values for $\epsilon_{b}, \epsilon_{c}$, and $\epsilon_{j}$ gives numerical values of roughly $0.48,0.09$, and 0.006 for $\epsilon_{4 j 2 \mathrm{btag}}^{c b c b}, \epsilon_{4 j 2 \mathrm{btag}}^{c b c s}$ and $\epsilon_{4 j 2 b t a g}^{c s c s}$, respectively. Note that the three terms in $\epsilon_{4 j 2 b t a g}^{c b c b}$ correspond to the cases of the two tagged $b$-jets originating from (i) two real $b$ quarks, (ii) one real $b$ quark and one fake $b$ quark (i.e., a mistagged $c$ quark), and (iii) two fake $b$ quarks. In $\epsilon_{4 j 2 \mathrm{btag}}^{c b c s}$, the first two terms correspond to the case of the two tagged $b$-jets originating from one real $b$ quark and one fake $b$ quark, and the last two terms are for the case of two fake $b$ quarks. In $\epsilon_{4 j 2 b t a g}^{c s c s}$, the only contributing terms are from two fake $b$ quarks. Factors of 2 or 4 in these expressions account for the various combinations that contribute (e.g., $c \bar{s}$ and $\bar{c} s$ being the fake $b$-tags in the third term in $\epsilon_{4 j 2 \mathrm{btag}}^{c b c s}$, leading to a factor of 2).

\section{Signal in four-jet channel with exactly one b-tagged jet}

The number of four-jet events in which exactly one of the jets is tagged as a $b$ quark is denoted by $S_{4 j 1 b t a g}$ and is given by the following expression:

$$
\begin{aligned}
S_{4 j \mathrm{lbtag}}= & \sigma \times \mathcal{L} \times \epsilon_{4 \mathrm{jnobtag}} \times\left(B R_{c b} B R_{c b} \epsilon_{4 j \mathrm{lbtag}}^{c b c b}\right. \\
& \left.+2 B R_{c b} B R_{c s} \epsilon_{4 j \mathrm{lbtag}}^{c b c s}+B R_{c s} B R_{c s} \epsilon_{4 j \mathrm{lbtag}}^{c s c s}\right) .
\end{aligned}
$$

The explicit expressions for $\epsilon_{4 j \text { lbtag }}^{c b c b}, \epsilon_{4 j \text { lbtag }}^{c b c s}$, and $\epsilon_{4 j \text { lbtag }}^{c s c s}$ (which are different to those for the two $b$-tag case) are as follows:

$$
\begin{aligned}
\epsilon_{4 j \mathrm{lbtag}}^{c b c b}= & 2 \epsilon_{b}\left(1-\epsilon_{b}\right)\left(1-\epsilon_{c}\right)^{2}+2\left(1-\epsilon_{b}\right)^{2} \epsilon_{c}\left(1-\epsilon_{c}\right), \\
\epsilon_{4 j \mathrm{btag}}^{c b c s} & =\epsilon_{b}\left(1-\epsilon_{c}\right)^{2}\left(1-\epsilon_{j}\right)+2\left(1-\epsilon_{b}\right) \epsilon_{c}\left(1-\epsilon_{c}\right)\left(1-\epsilon_{j}\right) \\
& +\epsilon_{j}\left(1-\epsilon_{b}\right)\left(1-\epsilon_{c}\right)^{2}, \\
\epsilon_{4 j \mathrm{btag}}^{c s c s} & =2 \epsilon_{j}\left(1-\epsilon_{c}\right)^{2}\left(1-\epsilon_{j}\right)+2 \epsilon_{c}\left(1-\epsilon_{c}\right)\left(1-\epsilon_{j}\right)^{2} .
\end{aligned}
$$

Inserting the values for $\epsilon_{b}, \epsilon_{c}$, and $\epsilon_{j}$ gives numerical values of roughly $0.38,0.65$, and 0.13 for $\epsilon_{4 j \mathrm{lbtag}}^{c b c b}, \epsilon_{4 j \mathrm{lbtag}}^{c b c s}$, and $\epsilon_{4 j 1 \mathrm{btag}}^{c s c}$, respectively.

\section{Signal in two-jet plus $\tau \nu$ channel with exactly one b-tagged jet}

The number of two-jet $+\tau \nu$ events in which exactly one of the jets is tagged as a $b$ quark is denoted by $S_{2 j \tau 1 \text { btag }}$ and is given by the following expression:

$$
\begin{aligned}
S_{2 j \tau \mathrm{lbtag}}= & \sigma \times \mathcal{L} \times \epsilon_{2 j \tau \mathrm{nobtag}} \times 2\left(B R_{c b} B R_{\tau \nu} \epsilon_{2 j \tau \mathrm{b} \mathrm{btag}}^{c b \nu \nu}\right. \\
& \left.+B R_{c s} B R_{\tau \nu} \epsilon_{2 j \tau \mathrm{lbtag}}^{c s \nu}\right) .
\end{aligned}
$$

The explicit expressions for $\epsilon_{2 j \tau \text { btag }}^{c b \tau \nu}$ and $\epsilon_{2 j \tau 1 \text { btag }}^{c s \tau \nu}$ are as follows:

$$
\begin{aligned}
& \epsilon_{2 j \tau 1 \mathrm{btag}}^{c b \tau \nu}=\epsilon_{b}\left(1-\epsilon_{c}\right)+\epsilon_{c}\left(1-\epsilon_{b}\right), \\
& \epsilon_{2 j \tau 1 \mathrm{btag}}^{c s \tau \nu}=\epsilon_{c}\left(1-\epsilon_{j}\right)+\epsilon_{j}\left(1-\epsilon_{c}\right) .
\end{aligned}
$$

The numerical values of $\epsilon_{2 j \tau \mathrm{bbtag}}^{c b \nu \nu}$ and $\epsilon_{2 j \tau \mathrm{b} b t a g}^{c s \tau \nu}$ are roughly 0.68 and 0.07 , respectively.

\section{B. Background to $\boldsymbol{H}^{ \pm} \rightarrow \boldsymbol{c b}$ decay}

The backgrounds for the above three channels are denoted by $B_{4 j 2 \mathrm{btag}}, B_{4 j 1 \mathrm{btag}}$, and $B_{2 j \tau 1 \mathrm{btag}}$, respectively. The main contributions to $B_{4 j 2 b t a g}$ and $B_{4 j 1 \text { btag }}$ are from four-fermion production (mainly $W^{+} W^{-}$production, with a 
smaller contribution from $Z Z$ ) and from two-fermion production (e.g., $e^{+} e^{-} \rightarrow \gamma^{*}, Z^{*} \rightarrow q \bar{q} g g$ ), which can give four jets. The main contribution to $B_{2 j \tau 1 \mathrm{btag}}$ is from $W^{+} W^{-}$ production. The $Z Z$ contribution to the four-fermion background will be neglected due to its cross section being about 25 times smaller than that of $W^{+} W^{-}$production (e.g., see [10]). It will be shown below that very few background events originate from $Z Z$ after $b$-tag requirements.

To evaluate the background before imposing $b$-tagging, we again use the numbers in the OPAL search paper [39]. For simplicity, we assume a diagonal CKM matrix and take $\mathrm{BR}\left(W^{ \pm} \rightarrow c s\right)=\mathrm{BR}\left(W^{ \pm} \rightarrow u d\right)=35 \%$. OPAL had around 1100 four-jet events after all cuts, of which $90 \%$ are expected to be from four-fermion events. With the assumption of a diagonal CKM matrix, this background would be composed of $250 \operatorname{cs} \bar{c} \bar{s}$ events, $250 u d \bar{u} \bar{d}$ events, and 500 csud events. Using the actual values for $\left|V_{u s}\right|^{2}$ and $\left|V_{c d}\right|^{2}$ [46], which are a factor of 20 times smaller than $\left|V_{c s}\right|^{2}$ and $\left|V_{u d}\right|^{2}$, would not change our numerical analysis. The decays $W^{ \pm} \rightarrow u d$ and $W^{ \pm} \rightarrow u s$ give the same contribution to the background (the same applies to $W^{ \pm} \rightarrow c s$ and $W^{ \pm} \rightarrow c d$ ) because $u, d$, and $s$ quarks all have the same efficiency for faking a $b$ quark. Given the above numbers, it turns out that the contributions to the background from $W^{ \pm} \rightarrow c b$ decays can be neglected because its branching ratio is about 550 times smaller than that of $W^{ \pm} \rightarrow c s$ [46], due to the small value of $\left|V_{c b}\right|$. The contribution of $W^{+} W^{-} \rightarrow c b \bar{c} \bar{b}$ to the background would be much less than one event $\left(=250 / 550^{2}\right)$, and the contributions from $W^{+} W^{-} \rightarrow$ cbcs and $W^{+} W^{-} \rightarrow$ cbud would each be less than one event $(=500 / 550)$, before $b$-tagging is imposed.

The $Z Z$ background is about 25 times less than that of the $W^{+} W^{-}$background and so one would expect around 40 $(=1000 / 25)$ four-fermion events to be from ZZ. However, in the channel with two $b$-tags (which in the case of the $H^{ \pm} \rightarrow c b$ signal would be tagged $b$-jets in opposite hemispheres), the suppression factor of $\epsilon_{b}^{2}[\operatorname{BR}(Z \rightarrow b \bar{b})]^{2}=$ $0.7^{2} \times 0.15^{2}$ would reduce this background to less than one event. The $Z Z$ backgrounds in the two $b$-tag and exactly one $b$-tag channels with $Z \rightarrow b b$ and $Z \rightarrow c \bar{c}$ are also negligible after multiplication by branching ratio factors, $\epsilon_{b}$ and $\epsilon_{c}$. Hereafter we neglect the $Z Z$ background.

\section{Background to four-jet channel with exactly two b-tagged jets}

The four-fermion background to the four-jet signal with two tagged $b$ quarks is given by

$$
\begin{aligned}
B_{4 j 2 \mathrm{btag}}^{4 \text { fermion }}= & 1000 \times\left(0.25 \times \epsilon_{4 j 2 \mathrm{btag}}^{W c s c s}+0.5 \times \epsilon_{4 j 2 \mathrm{btag}}^{W c s u d}\right. \\
& \left.+0.25 \times \epsilon_{4 j \text { btag }}^{\text {Wudud }}\right) .
\end{aligned}
$$

The explicit expressions for $\epsilon_{4 j 2 \mathrm{btag}}^{W c s c s}, \epsilon_{4 j 2 \mathrm{btag}}^{W c s u d}$, and $\epsilon_{4 j 2 \mathrm{btag}}^{W u d u d}$ are as follows: $\epsilon_{4 j 2 \mathrm{btag}}^{W c s c s}=4 \epsilon_{c} \epsilon_{j}\left(1-\epsilon_{c}\right)\left(1-\epsilon_{j}\right)+\epsilon_{c}^{2}\left(1-\epsilon_{j}\right)^{2}+\epsilon_{j}^{2}\left(1-\epsilon_{c}\right)^{2}$,

$\epsilon_{4 j \text { btag }}^{W c \text { sud }}=3 \epsilon_{c} \epsilon_{j}\left(1-\epsilon_{j}\right)^{2}+3 \epsilon_{j}^{2}\left(1-\epsilon_{c}\right)\left(1-\epsilon_{j}\right)$,

$\epsilon_{4 j 2 \mathrm{btag}}^{\text {Wudud }}=4 \epsilon_{j}^{2}\left(1-\epsilon_{j}\right)^{2}$.

The numerical values of $\epsilon_{4 j 2 \text { btag }}^{W c s c s}, \epsilon_{4 j 2 b t a g}^{W c s u d}$, and $\epsilon_{4 j 2 b \text { tag }}^{W u d u d}$ are $0.006,0.002$, and 0.0004 , respectively, giving $B_{4 j 2 \mathrm{btag}}^{4 \text { fermion }} \approx 2.6$.

OPAL [39] had around 100 four-jet events that originated from two-fermion events. Around 15 of these would be $b \bar{b}$ events, due to $\sigma\left(e^{+} e^{-} \rightarrow b \bar{b}\right) / \sigma\left(e^{+} e^{-} \rightarrow\right.$ $u \bar{u}, d \bar{d}, s \bar{s}, c \bar{c}, b \bar{b})$ being roughly 0.15 at $\sqrt{s}=200 \mathrm{GeV}$ [45]. We estimate the two-fermion background to the fourjet signal with two tagged $b$ quarks to be

$$
B_{4 j 2 \mathrm{btag}}^{2 \text { fermion }}=15 \epsilon_{b}^{2} \text {. }
$$

This is around seven events. The contribution to the twofermion background from $c \bar{c}$ events would be around $15 \epsilon_{c}^{2}$ and is much smaller than one event. The total background $\left(B_{4 j 2 \mathrm{btag}}\right)$ to the signal with four-jets and two tagged $b$ quarks $\left(S_{4 j 2 \mathrm{btag}}\right)$ is

$$
B_{4 j 2 \mathrm{btag}}=B_{4 j \text { btag }}^{4 \text { fermion }}+B_{4 j 2 \mathrm{btag}}^{2 \text { fermion }} .
$$

Since $B_{4 j 2 \mathrm{btag}}^{4 \text { fermion }}$ is around two events, then the dominant background is from the two-fermion events. The total background in this channel $\left(B_{4 j 2 \text { btag }}\right)$ is around 10 events.

\section{Background to four-jet channel with exactly one b-tagged jet}

The four-fermion background to the four-jet signal with one tagged $b$-jet is given by

$$
\begin{aligned}
B_{4 j \mathrm{lbtag}}^{4 \text { fermion }}= & 1000 \times\left(0.25 \times \epsilon_{4 j 1 \mathrm{btag}}^{W c s c s}+0.5 \times \epsilon_{4 j \mathrm{lbtag}}^{W c s u d}\right. \\
& \left.+0.25 \times \epsilon_{4 j \mathrm{btag}}^{W u d u d}\right) .
\end{aligned}
$$

The explicit expressions for $\epsilon_{4 j \text { lbtag }}^{W c s c s}, \epsilon_{4 j \text { lbtag }}^{W c s u d}$, and $\epsilon_{4 j \text { lbtag }}^{W u d u d}$ are as follows:

$$
\begin{aligned}
\epsilon_{4 j \text { btag }}^{W c s c s} & =2\left(1-\epsilon_{c}\right)^{2} \epsilon_{j}\left(1-\epsilon_{j}\right)+2 \epsilon_{c}\left(1-\epsilon_{c}\right)\left(1-\epsilon_{j}\right)^{2}, \\
\epsilon_{4 j \text { btag }}^{W c s u d} & =3 \epsilon_{j}\left(1-\epsilon_{c}\right)\left(1-\epsilon_{j}\right)^{2}+\epsilon_{c}\left(1-\epsilon_{j}\right)^{3}, \\
\epsilon_{4 j \text { lbtag }}^{W \text { udud }} & =4 \epsilon_{j}\left(1-\epsilon_{j}\right)^{3} .
\end{aligned}
$$

The numerical values of $\epsilon_{4 j \text { btag }}^{W c s c}, \epsilon_{4 j \text { lbtag }}^{W c s u}$, and $\epsilon_{4 j \text { lbtag }}^{\text {Wudud }}$ are $0.13,0.09$, and 0.04 , respectively, giving $B_{4 j \text { lbtag }}^{4 \text { fermion }} \approx 85$.

We estimate the two-fermion background (from $b \bar{b}$ production) to the four-jet signal with one tagged $b$ quark to be

$$
B_{4 j \mathrm{lbtag}}^{2 \text { fermion }}=30 \epsilon_{b}\left(1-\epsilon_{b}\right)
$$


This is about six events, but is much less than the fourfermion background, which is of the order of 85 events. We neglect the contribution to the two-fermion background from $c \bar{c}$ events, which would be $30 \epsilon_{c}\left(1-\epsilon_{c}\right)$ and equal to around 1.7 events. Similar to before, one has

$$
B_{4 j 1 \text { btag }}=B_{4 j \text { lbtag }}^{4 \text { fermion }}+B_{4 j \text { lbtag }}^{2 \text { fermion }} .
$$

The total background in this channel $\left(B_{4 j 1 \mathrm{btag}}\right)$ is around 91 events.

\section{Background to two-jet plus $\tau \nu$ channel with exactly one b-tagged jet}

The number of background events in the two-jet plus $\tau \nu$ channel in the OPAL [39] search is 316. Due to $\mathrm{BR}\left(W^{ \pm} \rightarrow\right.$ $c b)$ being about 600 times smaller than $\mathrm{BR}\left(W^{ \pm} \rightarrow c s\right)$ and $\operatorname{BR}\left(W^{ \pm} \rightarrow u d\right)$, there would be less than one event of $c b \tau \nu$ in the 316 OPAL events of two-jet plus $\tau \nu$. Hence, this contribution is neglected. The background to the two-jet plus $\tau \nu$ channel with exactly one $b$-tagged jet is dominantly from four-fermion production with a fake $b$ quark and is given by

$$
B_{2 j \tau \text { lbtag }}^{4 \text { fermion }}=316 \times \frac{1}{2} \times\left(\epsilon_{2 j \tau \text { lbtag }}^{W c s \tau \nu}+\epsilon_{2 j \tau \text { lbtag }}^{W u d \tau \nu}\right) .
$$

The explicit expressions for $\epsilon_{2 j \tau 1 \text { btag }}^{W c s \tau}$ and $\epsilon_{2 j \tau 1 \text { btag }}^{W u d \tau \nu}$ are as follows:

$$
\begin{aligned}
& \epsilon_{2 j \tau 1 \mathrm{btag}}^{W c s \tau \nu}=\epsilon_{c}\left(1-\epsilon_{j}\right)+\epsilon_{j}\left(1-\epsilon_{c}\right) . \\
& \epsilon_{2 j \tau \mathrm{lbtag}}^{W u d \tau}=2 \epsilon_{j}\left(1-\epsilon_{j}\right) .
\end{aligned}
$$

The numerical values of $\epsilon_{2 j \tau 1 \mathrm{ltag}}^{W c s \tau \nu}$ and $\epsilon_{2 j \tau \mathrm{lbtag}}^{W u d \tau \nu}$ are 0.07 and 0.02 , respectively. The total background in this channel $\left(B_{2 j \tau \text { lbtag }}^{4 \text { fermion }}\right)$ is around 14 events.

We now mention other production and decay channels of charged and neutral scalars (e.g., $C P$-even $h^{0}$ and $C P$-odd $A^{0}$ ) in a 3 HDM that might resemble the proposed signatures from $H^{ \pm} \rightarrow c b$. The production process $e^{+} e^{-} \rightarrow$ $h^{0} A^{0}$ was searched for at LEP2 [47] and gives rise to final states with two or four $b$ quarks. These signatures are different to the ones studied in this work (e.g., the two $b$ quarks from $h^{0}, A^{0} \rightarrow b \bar{b}$ are in the same hemisphere, and $M_{h^{0}}=125 \mathrm{GeV}$ ), and no evidence was found for them. This is accommodated in the 3HDM by taking the mass of the lightest $C P$-odd scalar $\left(M_{A_{1}^{0}}\right)$, which one of the 16 free parameters, to be greater than around $75 \mathrm{GeV}$. At a future $e^{+} e^{-}$collider with $\sqrt{s}=240 \mathrm{GeV}$ (to be discussed in Sec. IV B), the $e^{+} e^{-} \rightarrow h^{0} A_{1}^{0}$ channel could be used to probe $M_{A_{1}^{0}}$ between 75 and $115 \mathrm{GeV}$. Its experimental signature would be different to that from $H^{ \pm} \rightarrow c b$ (as mentioned above), and if $M_{A_{1}^{0}}>115 \mathrm{GeV}$, then there would be no events from $e^{+} e^{-} \rightarrow h^{0} A_{1}^{0}$.
The decay channel $H^{ \pm} \rightarrow A^{0} W^{*}$ (which can be dominant if $|X|,|Y|$, and $|Z|$ are small) was searched for at LEP2 in [43]. This channel gives rise to two or four $b$ quarks from $A^{0} \rightarrow b \bar{b}$. Regions in the plane $\left[M_{H^{ \pm}}, \tan \beta\right]$ for a given $M_{A^{0}}$ were excluded in the context of the 2HDM (Model I). Considerable sensitivity to the region $80 \mathrm{GeV} \leq M_{H^{ \pm}} \leq$ $90 \mathrm{GeV}$ was achieved. The signatures from $H^{ \pm} \rightarrow A^{0} W^{*}$ are different to those in this work; e.g., $A^{0} \rightarrow b \bar{b}$ for a light $A^{0}$ differs from $H^{ \pm} \rightarrow c b$. Given that no evidence for a signal was found in either of the above searches, we conclude that these production processes would have little or no effect on our proposed search for $H^{ \pm} \rightarrow c b$. In this work, we assume that the decay $H^{ \pm} \rightarrow A^{0} W^{*}$ is not open.

Regarding the likelihood of the above searches for $H^{ \pm} \rightarrow c b$ being carried out, we note that some LEP data are still being analyzed and submitted for publication, e.g., $[48,49]$. We assume that if there is sufficient motivation for carrying out a new search with LEP data, then it can be done in principle. If there were an excess in the region $80 \mathrm{GeV} \leq M_{H^{ \pm}} \leq 90 \mathrm{GeV}$ in future searches for $t \rightarrow H^{ \pm} b$ with $H^{ \pm} \rightarrow c b$ at the LHC, then a search for $e^{+} e^{-} \rightarrow$ $H^{+} H^{-}$with $H^{ \pm} \rightarrow c b$ with LEP data would be very well motivated. Such a search is also motivated from the point of view of having sensitivity to the parameter space of very small $|X|$ and $|Y|$, for which searches at the LHC are ineffective (as mentioned in Sec. II).

\section{NUMERICAL RESULTS}

We now present our results for the statistical significances of a signal for $H^{ \pm} \rightarrow c b$ at LEP2 $(189 \mathrm{GeV} \leq \sqrt{s} \leq$ $209 \mathrm{GeV})$ and at CEPC/FCC-ee $(\sqrt{s}=240 \mathrm{GeV})$. In the context of LEP2, the region $80 \mathrm{GeV} \leq M_{H^{ \pm}} \leq 90 \mathrm{GeV}$ is studied, while at CEPC/FCC-ee we consider $80 \mathrm{GeV} \leq$ $M_{H^{ \pm}} \leq 120 \mathrm{GeV}$. Of the five types of 3HDM, the parameter space for a large $\operatorname{BR}\left(H^{ \pm} \rightarrow c b\right)$ is greatest in the flipped $3 \mathrm{HDM}$, and hence results are shown in this model only. In our numerical analysis at CEPC/FCC-ee, $\epsilon_{c}$ is varied in the range $0.01<\epsilon_{c}<0.06$, while $\epsilon_{b}$ and $\epsilon_{j}$ are conservatively taken to have the same values as at LEP2. Each LEP2 experiment accumulated around $0.6 \mathrm{fb}^{-1}$ of integrated luminosity $(\mathcal{L})$, while at CEPC/FCC-ee at least $1000 \mathrm{fb}^{-1}$ is expected. These input parameters are summarized in Table III.

\section{A. Enhancing the detection prospects for $H^{ \pm} \rightarrow c b$ at LEP2 by using $b$-tags}

The BRs of $H^{ \pm}$as functions of the four parameters $(\tan \beta, \tan \gamma, \theta, \delta)$ have been studied in detail in [21], and the parameter space for a dominant $\operatorname{BR}\left(H^{ \pm} \rightarrow c b\right)>50 \%$ was displayed. In Fig. 1 (left panel), contours of $\mathrm{BR}\left(H^{ \pm} \rightarrow c b\right)$ are shown in the plane $[\tan \gamma, \tan \beta]$, for $M_{H^{ \pm}}=80 \mathrm{GeV}$ (the results with $M_{H^{ \pm}}=89 \mathrm{GeV}$ are essentially identical). We fix $\theta=-\frac{\pi}{2.1}$ and $\delta=0$, for which a sizeable part of the 
TABLE III. Input parameters used in the numerical analysis at LEP2 and at CEPC/FCC-ee.

\begin{tabular}{lcccccc}
\hline \hline & $\sqrt{s}$ & $\mathcal{L}\left(\mathrm{fb}^{-1}\right)$ & $\epsilon_{b}$ & $\epsilon_{c}$ & $\epsilon_{j}$ & $M_{H^{ \pm}}$ \\
\hline LEP2 & $189 \mathrm{GeV} \rightarrow 209 \mathrm{GeV}$ & 0.6 & 0.7 & 0.06 & 0.01 & $80 \mathrm{GeV}<M_{H^{ \pm}}<90 \mathrm{GeV}$ \\
CEPC/FCC-ee & $240 \mathrm{GeV}$ & 1000 & 0.7 & $0.01<\epsilon_{c}<0.06$ & 0.01 & $80 \mathrm{GeV}<M_{H^{ \pm}}<120 \mathrm{GeV}$ \\
\hline \hline
\end{tabular}

plane $[\tan \gamma, \tan \beta]$ gives $\operatorname{BR}\left(H^{ \pm} \rightarrow c b\right)>60 \%$, with around $80 \%$ being the maximum value. Similar plots (for different choices of $\theta$ ) can be found in [21]. This parameter choice for $\theta$ and $\delta$ will be used in Figs. 1-7, with all these plots being shown in the plane $[\tan \gamma, \tan \beta]$. In Fig. 1 (right panel), contours of $\operatorname{Re}\left(X Y^{*}\right)$ are shown, with the region $-1.1 \leq \operatorname{Re}\left(X Y^{*}\right) \leq 0.7$ being (roughly) consistent with the limits on $\operatorname{BR}(b \rightarrow s \gamma)$ for $M_{H^{ \pm}}=80 \mathrm{GeV}$. Clearly, the majority of the plane $[\tan \gamma, \tan \beta]$ satisfies this constraint, and thus the large values of $\operatorname{BR}\left(H^{ \pm} \rightarrow c b\right)$ in Fig. 1 (left panel) are permissible. As discussed in [21], if the contribution of the heavier $H^{\prime \pm}$ in a $3 \mathrm{HDM}$ is not neglected, then the region of the plane $[\tan \gamma, \tan \beta]$ that satisfies the $b \rightarrow s \gamma$ constraint would shift with respect to that shown in Fig. 1 (right panel). This is because the couplings $\operatorname{Re}\left(X Y^{*}\right)$ for the heavier charged scalar would now enter the constraint, and these couplings also depend on the input parameters $\tan \gamma, \tan \beta, \theta$, and $\delta$. To determine the exact region in the plane $[\tan \gamma, \tan \beta]$ that is excluded by $b \rightarrow s \gamma$ would require a detailed numerical study (to at least next-to-leading order in the effective Lagrangian for $b \rightarrow s \gamma$ ), which is beyond the scope of this work. However, given the large region of $[\tan \gamma, \tan \beta]$ that is permissible in Fig. 1 (right panel), we expect that there would still be a sizeable region for large $\operatorname{BR}\left(H^{ \pm} \rightarrow c b\right)$ when the contribution of $H^{\prime \pm}$ is not neglected in the $b \rightarrow s \gamma$ constraint.

In Fig. 2 (left panel and right panel), contours of $\mathrm{BR}\left(H^{ \pm} \rightarrow c s\right)$ and $\quad \mathrm{BR}\left(H^{ \pm} \rightarrow \tau \nu\right) \quad$ (respectively) are

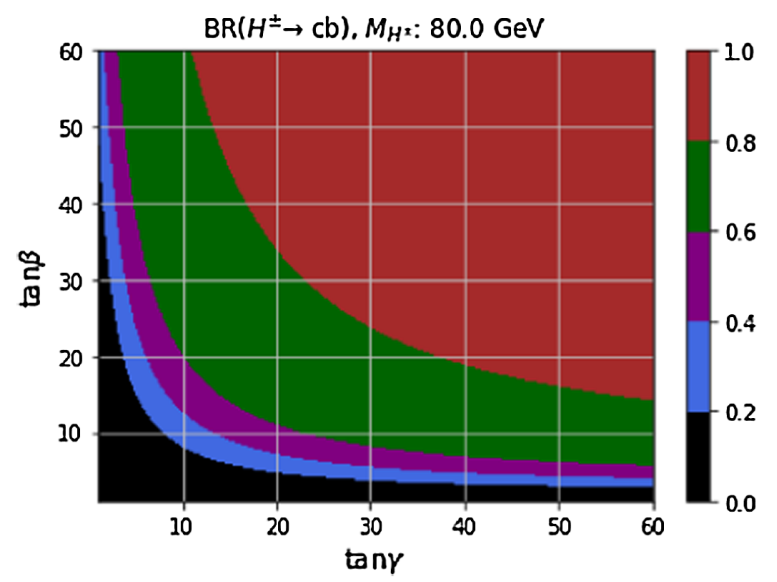

displayed. For this choice of $\theta=-\frac{\pi}{2.1}$ and $\delta=0$, one can see that $\operatorname{BR}\left(H^{ \pm} \rightarrow c s\right) \approx 35 \%$ and $\operatorname{BR}\left(H^{ \pm} \rightarrow \tau \nu\right) \approx$ $65 \%$ when $\operatorname{BR}\left(H^{ \pm} \rightarrow c b\right)$ is small (corresponding to small $\tan \beta$ and $\tan \gamma$ ). In Fig. 3, the sums and products of BRs of $H^{ \pm}$are displayed, which will aid in understanding of the statistical significances that are displayed in Figs. 4-7. In Fig. 3 (top left panel), contours of $\mathrm{BR}\left(H^{ \pm} \rightarrow\right.$ hadrons) are shown, where hadrons refer to the sum of $c s$ and $c b$. In Fig. 3 (top right panel and bottom panel), contours of $\mathrm{BR}\left(H^{ \pm} \rightarrow\right.$ hadrons $) \times \mathrm{BR}\left(H^{ \pm} \rightarrow \tau \nu\right)$ and $\mathrm{BR}\left(H^{ \pm} \rightarrow\right.$ $c b) \times \mathrm{BR}\left(H^{ \pm} \rightarrow \tau \nu\right)$ (respectively) are shown. Note that $\mathrm{BR}\left(H^{ \pm} \rightarrow c b\right) \times \mathrm{BR}\left(H^{ \pm} \rightarrow \tau \nu\right)$ is maximized (taking a value of around 0.14 ) in a band that is away from the region of both $\tan \beta$ and $\tan \gamma$ being small or large.

In Figs. 4-7, the statistical significances $(S / \sqrt{B})$ which could have been obtained at a single experiment at LEP2 are shown in the five channels (three with $b$-tagging and two without $b$-tagging) in the plane $[\tan \gamma, \tan \beta]$ for $M_{H^{ \pm}}=80$ and $89 \mathrm{GeV}$. In the three panels in Fig. 4, $S_{4 \text { jnobtag }} / \sqrt{B_{4 \text { jnobtag }}}$ for the four-jet channel (top panel), $S_{4 j 2 \mathrm{btag}} / \sqrt{B_{4 j 2 \mathrm{btag}}}$ (left bottom panel), and $S_{4 j \mathrm{lbtag}} /$ $\sqrt{B_{4 j \text { lbtag }}}$ (right bottom panel) are plotted, with $M_{H^{ \pm}}=$ $80 \mathrm{GeV}$. For the case with no $b$-tagging (which corresponds to the experimental searches), one can see that the largest signal satisfies $2<S / \sqrt{B}<2.5$ and arises in the region where $\mathrm{BR}\left(H^{ \pm} \rightarrow\right.$ hadrons) in Fig. 3 (top left panel) is largest. The maximum $S / \sqrt{B}$ is less than 2.5 for this choice of $M_{H^{ \pm}}=80 \mathrm{GeV}$, and this is roughly consistent

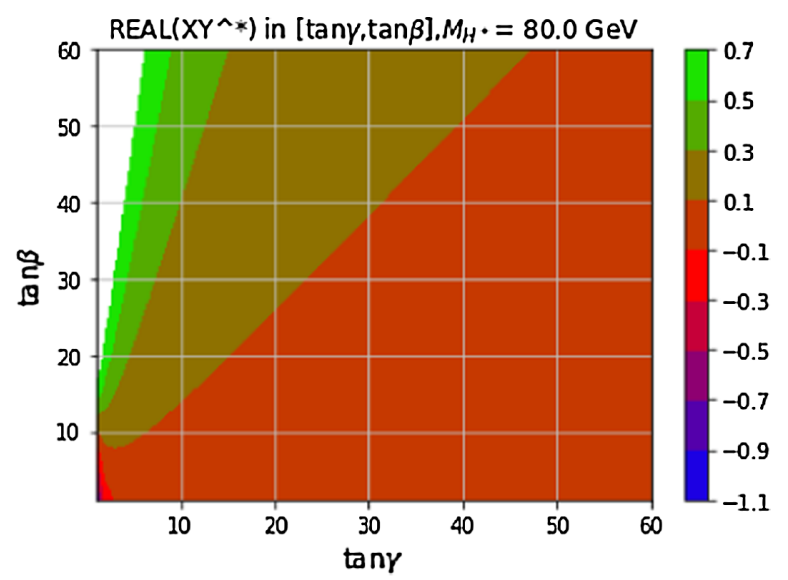

FIG. 1. The flipped 3HDM with $\theta=-\frac{\pi}{2.1}, \delta=0$, and $M_{H^{ \pm}}=80 \mathrm{GeV}$. Left panel: contours of $\mathrm{BR}\left(H^{ \pm} \rightarrow c b\right)$ in the plane $[\tan \gamma, \tan \beta]$. Right panel: contours of $\operatorname{Re}\left(X Y^{*}\right)$ where the region $-1.1 \leq \operatorname{Re}\left(X Y^{*}\right) \leq 0.7$ is consistent with the limits on $\mathrm{BR}(b \rightarrow s \gamma)$ for $M_{H^{ \pm}}=80 \mathrm{GeV}$. 

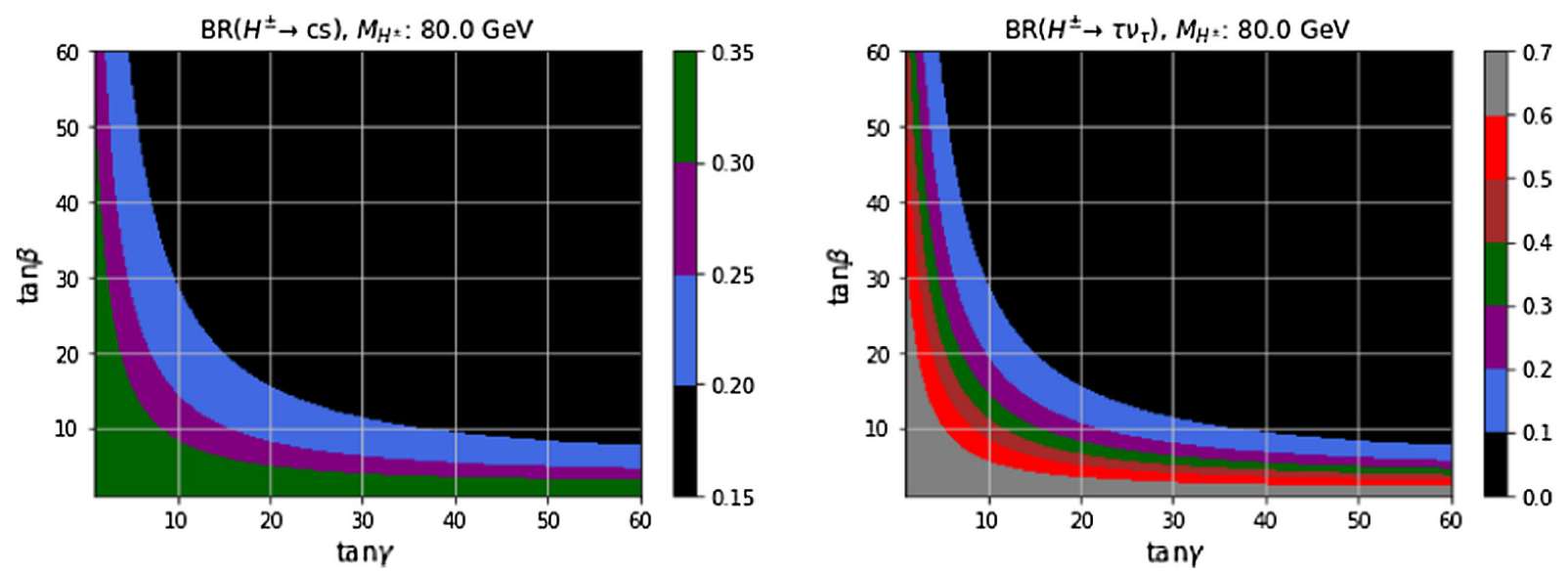

FIG. 2. The flipped $3 \mathrm{HDM}$ with $\theta=-\frac{\pi}{2.1}, \delta=0$, and $M_{H^{ \pm}}=80 \mathrm{GeV}$. Left panel: contours of $\mathrm{BR}\left(H^{ \pm} \rightarrow c s\right)$ in the plane $[\tan \gamma, \tan \beta]$. Right panel: contours of $\operatorname{BR}\left(H^{ \pm} \rightarrow \tau \nu\right)$ in the plane $[\tan \gamma, \tan \beta]$.

with the OPAL limits that ruled out $M_{H^{ \pm}}<80 \mathrm{GeV}$ for $\operatorname{BR}\left(H^{ \pm} \rightarrow\right.$ hadrons $)=100 \%$. For the case with two $b$-tags, it is evident that $S / \sqrt{B}$ can be greatly increased with respect to the case with no $b$-tag. A large part of the

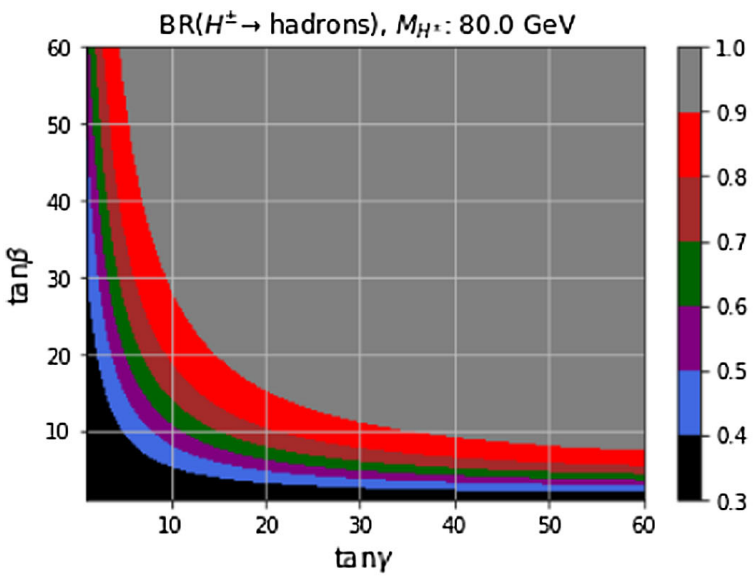

$[\tan \gamma, \tan \beta]$ plane has $S / \sqrt{B}>3$, with $S / \sqrt{B} \approx 8$ being possible. Note that these significances are for a single LEP2 experiment, and thus a $3 \sigma$ signal at all four experiments might approach the $5 \sigma$ threshold for discovery if the four
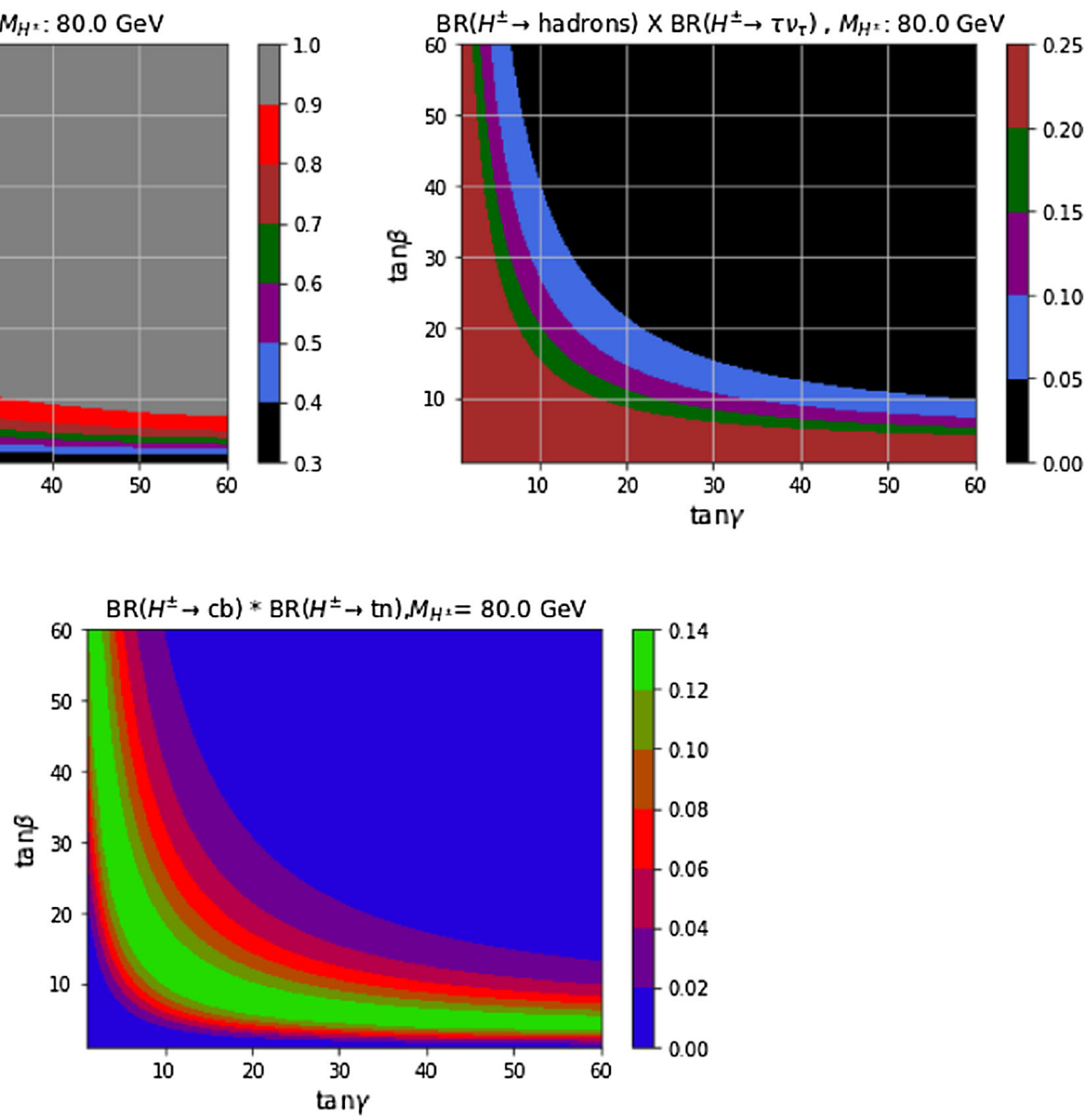

FIG. 3. The flipped 3HDM with $\theta=-\frac{\pi}{2.1}, \delta=0$, and $M_{H^{ \pm}}=80 \mathrm{GeV}$. Top left panel: contours of $\mathrm{BR}\left(H^{ \pm} \rightarrow\right.$ hadrons $)$ in the plane $[\tan \gamma, \tan \beta]$, where "hadrons" refers to the sum of $c s$ and $c b$. Top right panel: contours of $\operatorname{BR}\left(H^{ \pm} \rightarrow \operatorname{hadrons}\right) \times \mathrm{BR}\left(H^{ \pm} \rightarrow \tau \nu\right)$ in the plane $[\tan \gamma, \tan \beta]$. Bottom panel: contours of $\operatorname{BR}\left(H^{ \pm} \rightarrow c b\right) \times \mathrm{BR}\left(H^{ \pm} \rightarrow \tau \nu\right)$ in the plane $[\tan \gamma, \tan \beta]$. 

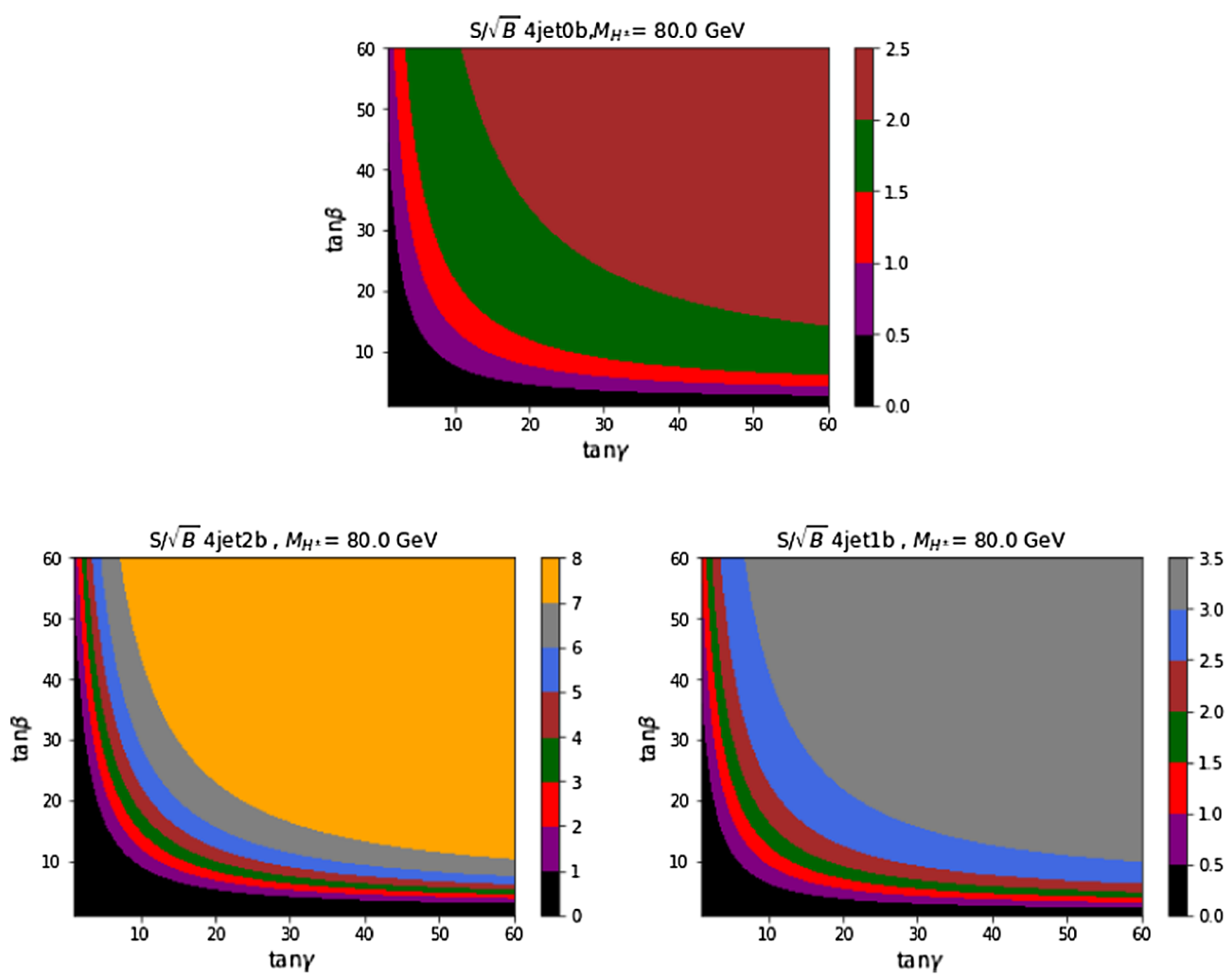

FIG. 4. The flipped $3 \mathrm{HDM}$ with $\theta=-\frac{\pi}{2.1}, \delta=0$, and $M_{H^{ \pm}}=80 \mathrm{GeV}$. Top panel: significance $(S / \sqrt{B})$ at a single LEP2 experiment in the four-jet channel without $b$-tagging in $[\tan \gamma, \tan \beta]$ plane. Left bottom panel: $S / \sqrt{B}$ in the four-jet channel with two $b$-tags in $[\tan \gamma, \tan \beta]$ plane. Right bottom panel: $S / \sqrt{B}$ in the four-jet channel with one $b$-tag in $[\tan \gamma, \tan \beta]$ plane.

searches are combined. The individual values of $S$ and $B$ will be shown in tables and discussed later. For the case with one $b$-tag, it is found that the values of $S / \sqrt{B}$ (at a given point in the plane) are slightly larger than those for the case with no $b$-tag. Although the background in the one $b$-tag channel is smaller, the signal has decreased such that

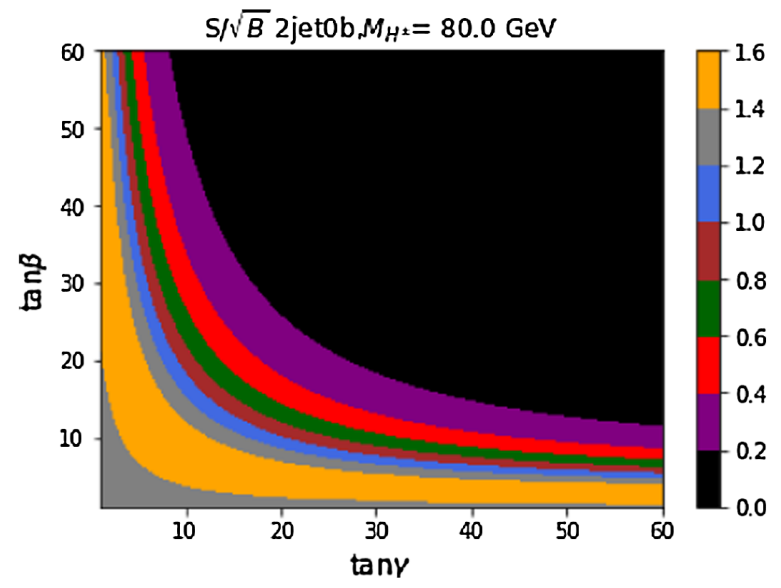

(a) the ratio $S / \sqrt{B}$ does not improve greatly compared to the case with no $b$-tag.

In the two panels in Fig. 5, $S_{2 j \text { nnobtag }} / \sqrt{B_{2 j \text { nobtag }}}$ for the two-jet channel (left panel) and $S_{2 j \tau \mathrm{lbtag}} / \sqrt{B_{2 j \tau \mathrm{lbtag}}}$ (right panel) are plotted, with $M_{H^{ \pm}}=80 \mathrm{GeV}$. For the case with

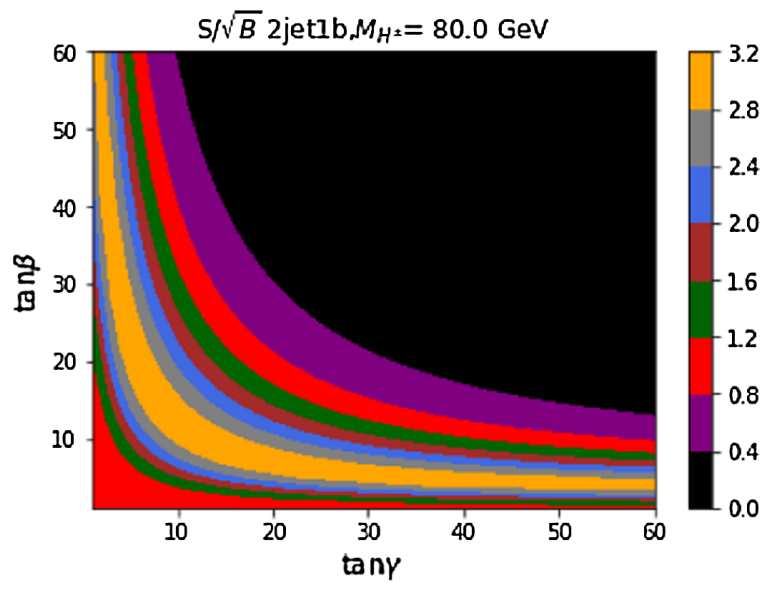

(b)

FIG. 5. The flipped 3HDM with $\theta=-\frac{\pi}{2.1}, \delta=0$, and $M_{H^{ \pm}}=80 \mathrm{GeV}$. Left panel: significance $(S / \sqrt{B})$ at a single LEP2 experiment in the two-jet channel without $b$-tagging in $[\tan \gamma, \tan \beta]$ plane. Right panel: $S / \sqrt{B}$ in the two-jet channel with one $b$-tag in $[\tan \gamma, \tan \beta]$ plane. 

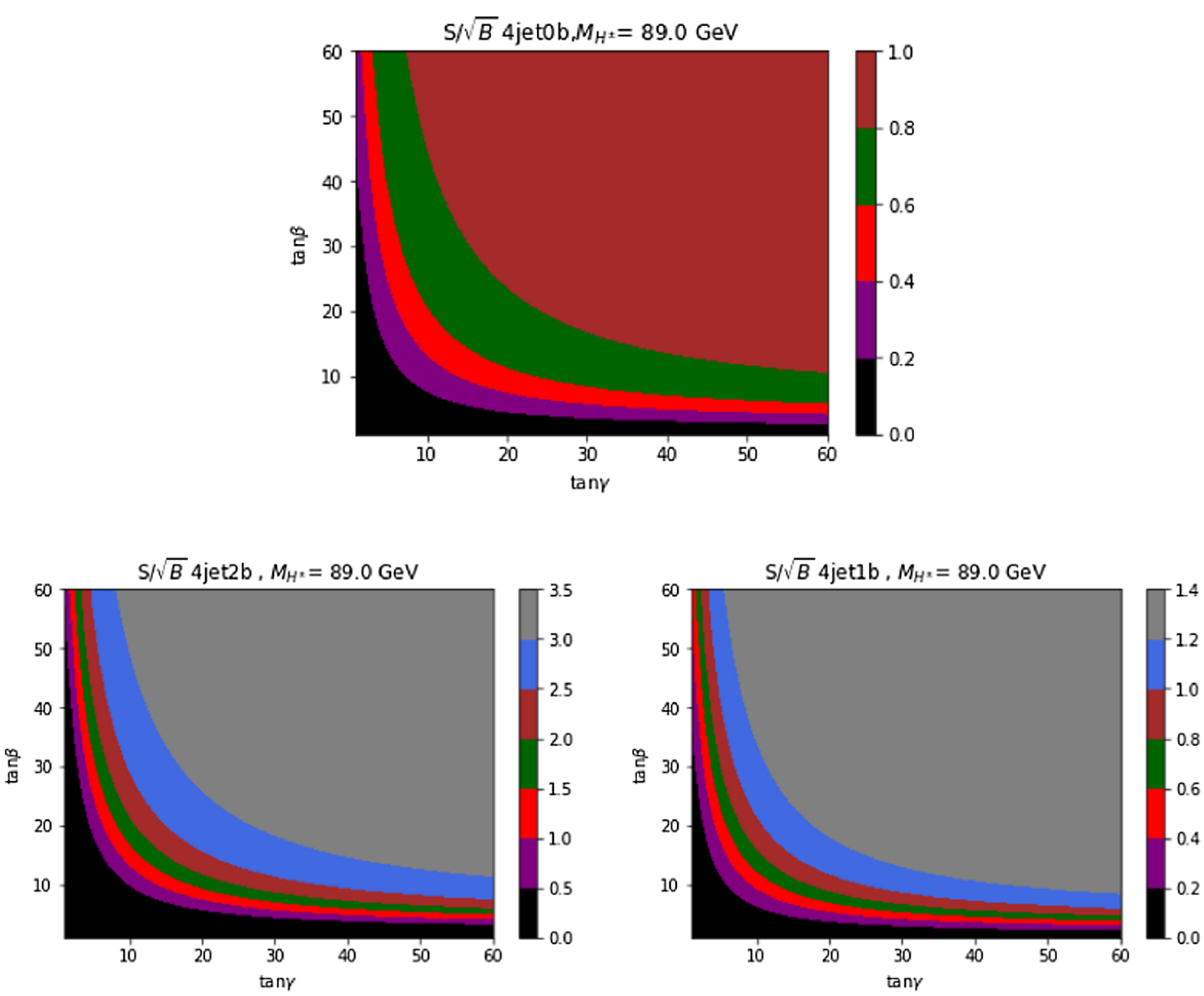

FIG. 6. The flipped 3HDM with $\theta=-\frac{\pi}{2.1}, \delta=0$, and $M_{H^{ \pm}}=89 \mathrm{GeV}$. Top panel: significance $(S / \sqrt{B})$ at a single LEP2 experiment in the four-jet channel without $b$-tagging in $[\tan \gamma, \tan \beta]$ plane. Left bottom panel: $S / \sqrt{B}$ in the four-jet channel with two $b$-tags in $[\tan \gamma, \tan \beta]$ plane. Right bottom panel: $S / \sqrt{B}$ in the four-jet channel with one $b$-tag in $[\tan \gamma, \tan \beta]$ plane.

no $b$-tagging, one sees that the largest $S / \sqrt{B}$ is around 1.6 and arises in the region where $\operatorname{BR}\left(H^{ \pm} \rightarrow\right.$ hadrons $) \times$ $\operatorname{BR}\left(H^{ \pm} \rightarrow \tau \nu\right)$ in Fig. 3 (top right panel) is largest. Again, this maximum value for $S / \sqrt{B}$ is roughly consistent with the OPAL limits that ruled out $M_{H^{ \pm}}<80 \mathrm{GeV}$ [39] in the two-jet channel when $\operatorname{BR}\left(H^{ \pm} \rightarrow\right.$ hadrons $) \times$ $\operatorname{BR}\left(H^{ \pm} \rightarrow \tau \nu\right)$ is at its maximum value. For the case with one $b$-tag, it is evident that $S / \sqrt{B}$ can be somewhat increased with respect to the case with no $b$-tag, but the gain is less than that in the four-jet channel with two $b$-tags. Values of $S / \sqrt{B}$ up to 3.2 can be obtained in the region in Fig. 3 (bottom panel) where $\operatorname{BR}\left(H^{ \pm} \rightarrow c b\right) \times \mathrm{BR}\left(H^{ \pm} \rightarrow\right.$ $\tau \nu)$ is largest.

Figures 6 and 7 are the same as Figs. 4 and 5, respectively, but with $M_{H^{ \pm}}=89 \mathrm{GeV}$ instead of $80 \mathrm{GeV}$. The maximum $S / \sqrt{B}$ has dropped by roughly a factor of 2 for the four-jet channel with two $b$-tags and for the two-jet channel with one $b$-tag. This decrease is due to the reduction in the cross section for $e^{+} e^{-} \rightarrow H^{+} H^{-}$when going from $M_{H^{ \pm}}=$ $80 \mathrm{GeV}$ to $M_{H^{ \pm}}=89 \mathrm{GeV}$. As mentioned earlier, a $3 \sigma$ signal at each LEP2 experiment might become close to $5 \sigma$ evidence by combining all four experiments. Hence, a discovery for $M_{H^{ \pm}}=89 \mathrm{GeV}$ is possible in the most optimistic scenario of $\mathrm{BR}\left(H^{ \pm} \rightarrow c b\right)$ close to $80 \%$.

In Fig. 8, the dependence of $S / \sqrt{B}$ on $M_{H^{ \pm}}$is shown for the four-jet channel, fixing $\operatorname{BR}\left(H^{ \pm} \rightarrow c b\right)=0.8$ (i.e., near maximal) and $\operatorname{BR}\left(H^{ \pm} \rightarrow c s\right)=0.2$. The top panel, left bottom panel, and right bottom panel are for the channels without $b$-tagging, two $b$-tags, and one $b$-tag, respectively. One can see that the dependence is roughly linear, and that a $5 \sigma$ signal at a single LEP2 experiment is possible in the four-jet channel with two $b$-tags up to around $M_{H^{ \pm}}=84 \mathrm{GeV}$.

In Fig. 9, the dependence of $S / \sqrt{B}$ on $M_{H^{ \pm}}$is shown for the two-jet channel, with $\operatorname{BR}\left(H^{ \pm} \rightarrow c s\right)=0.1$, 


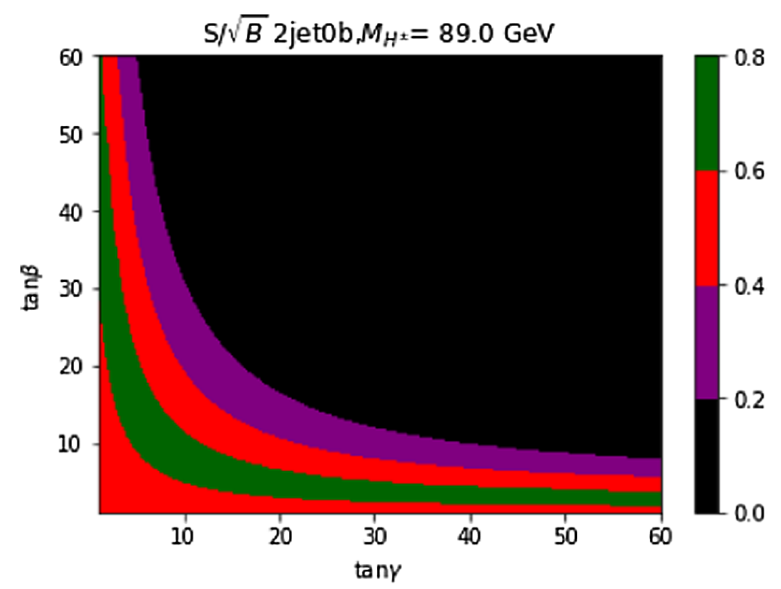

(a)

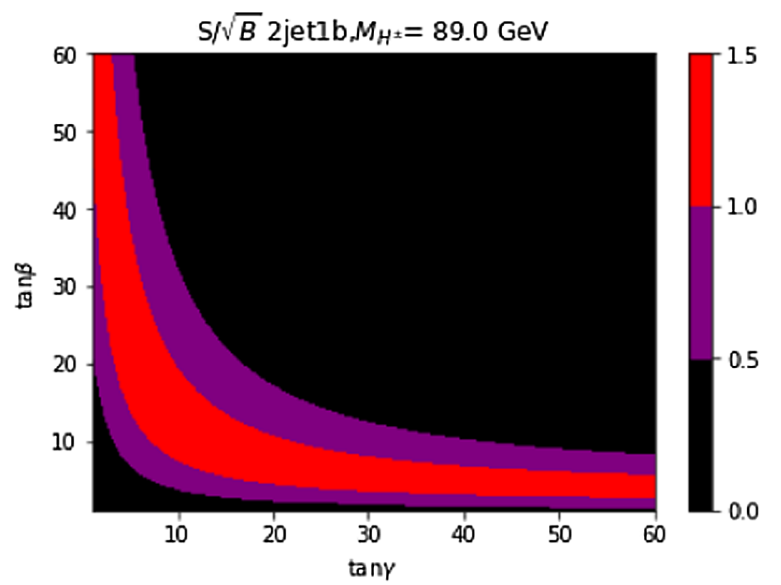

(b)

FIG. 7. The flipped 3HDM with $\theta=-\frac{\pi}{2.1}, \delta=0$, and $M_{H^{ \pm}}=89 \mathrm{GeV}$. Left panel: significance $(S / \sqrt{B})$ at a single LEP2 experiment in the two-jet channel without $b$-tagging in $[\tan \gamma, \tan \beta]$ plane. Right panel: $S / \sqrt{B}$ in the two-jet channel with one $b$-tag in $[\tan \gamma, \tan \beta]$ plane.

$\mathrm{BR}\left(H^{ \pm} \rightarrow c b\right)=0.4$, and $\mathrm{BR}\left(H^{ \pm} \rightarrow \tau \nu\right)=0.5$ (i.e., close to the optimum scenario for discovery in this channel). Note that this choice of $\operatorname{BR}\left(H^{ \pm} \rightarrow c b\right) \times \operatorname{BR}\left(H^{ \pm} \rightarrow \tau \nu\right)=$ 0.2 is used for illustration and is larger than the maximum value of this product in Fig. 3 (bottom panel) with $\theta=$ $-\pi / 2.1$ and $\delta=0$. Again, one sees a roughly linear dependence on $M_{H^{ \pm}}$. In Fig. $10, S / \sqrt{B}$ is plotted in the plane $\left[M_{H^{ \pm}}, \mathrm{BR}\left(H^{ \pm} \rightarrow c b\right)\right]$. In the left panel, we show the
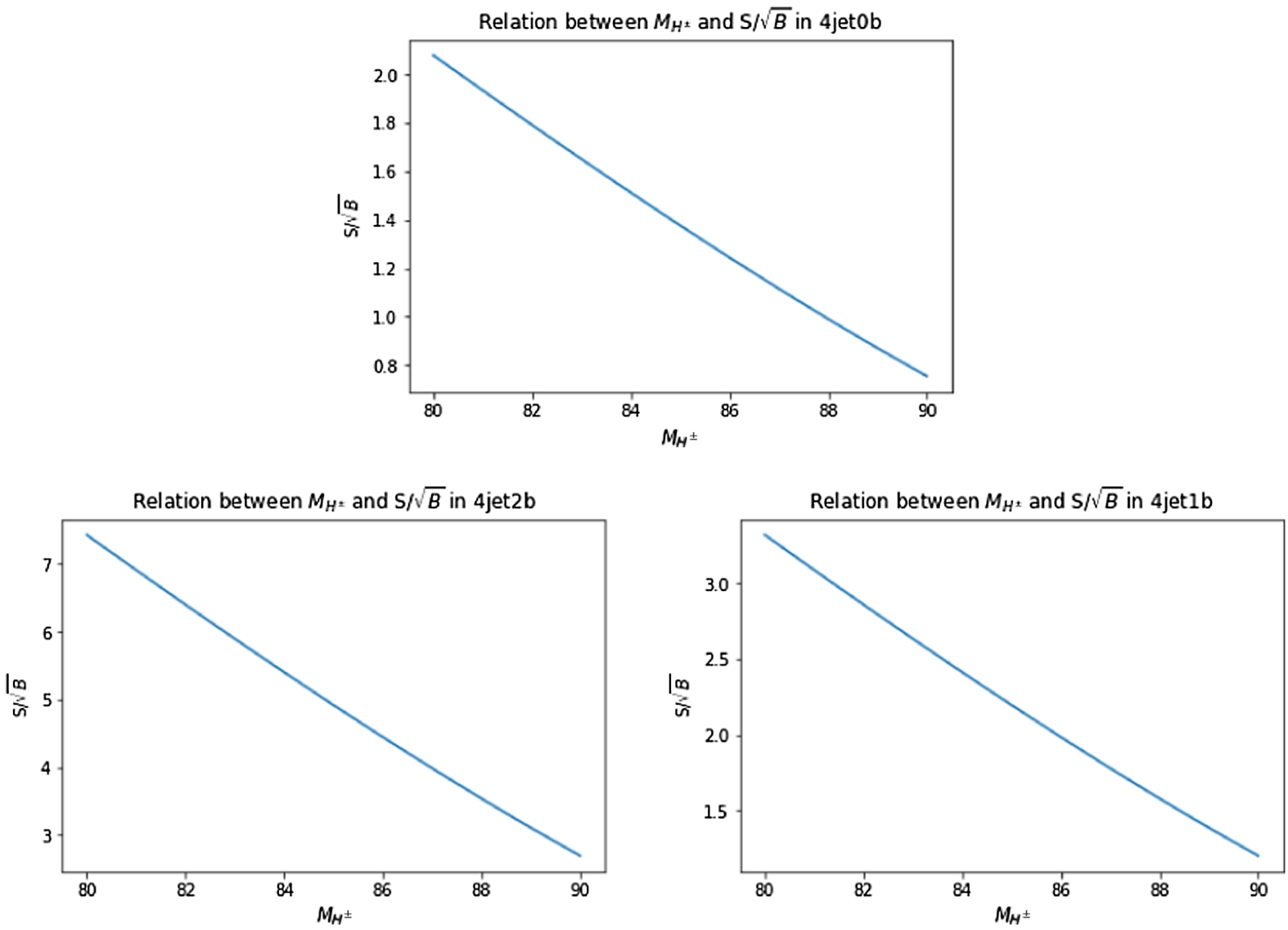

FIG. 8. Dependence of $S / \sqrt{B}$ on $M_{H^{ \pm}}$, with $\mathrm{BR}\left(H^{ \pm} \rightarrow c b\right)=0.8$ (near maximal) and $\mathrm{BR}\left(H^{ \pm} \rightarrow c s\right)=0.2$ at a single LEP2 experiment. Top panel: in four-jet channel without $b$-tagging. Left bottom panel: In four-jet channel with two $b$-tags. Right bottom panel: in 4-jet channel with one $b$-tag. 

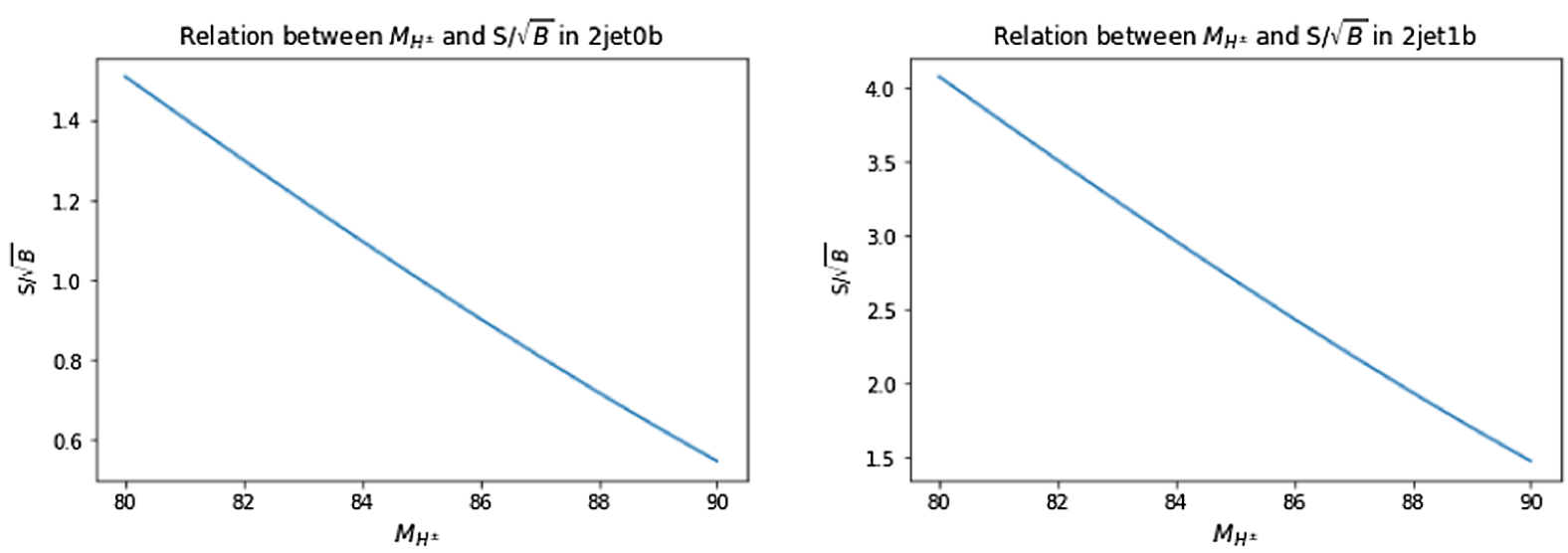

FIG. 9. Dependence of $S / \sqrt{B}$ on $M_{H^{ \pm}}$with $\mathrm{BR}\left(H^{ \pm} \rightarrow\right.$ hadrons $)=0.5$ and $\mathrm{BR}\left(H^{ \pm} \rightarrow \tau \nu\right)=0.5$ at a single LEP2 experiment. Left panel: in two-jet channel without $b$-tagging. Right panel: in two-jet channel with one $b$-tag.
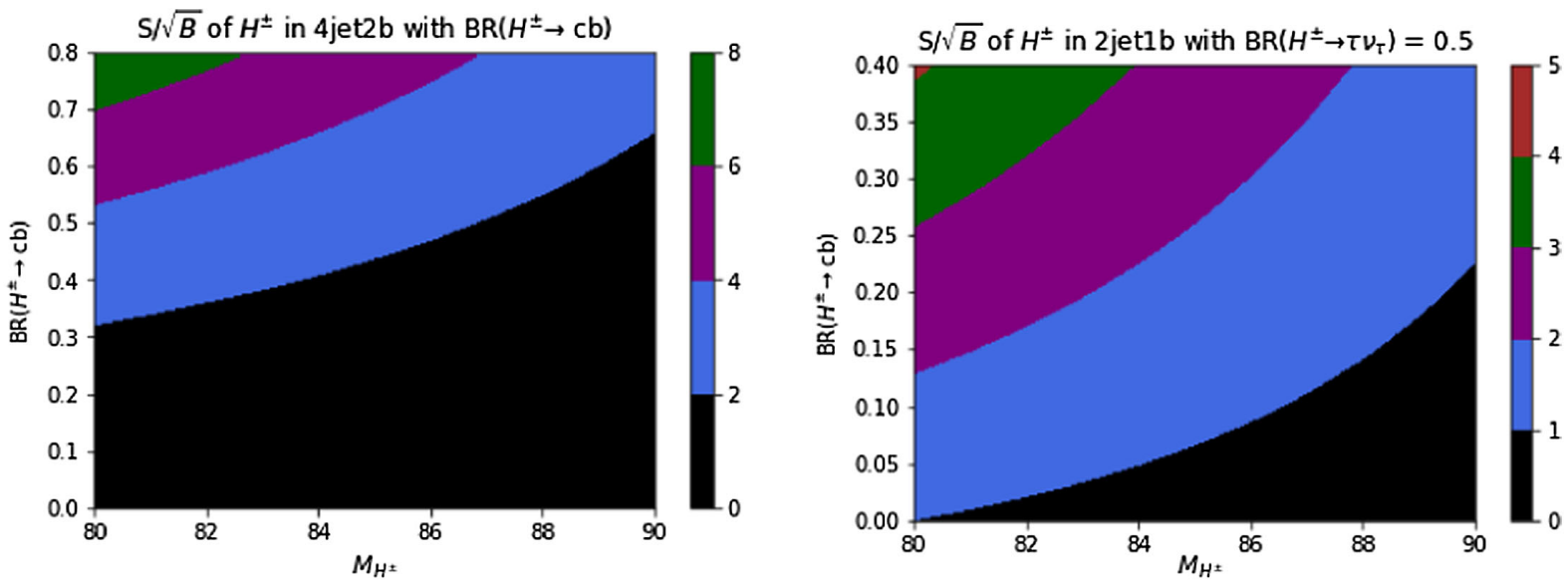

FIG. 10. Values of $S / \sqrt{B}$ in the plane $\left[M_{H^{ \pm}}, \mathrm{BR}\left(H^{ \pm} \rightarrow c b\right)\right]$ at a single LEP2 experiment. Left panel: in four-jet channel with two $b$-tags with $\mathrm{BR}\left(H^{ \pm} \rightarrow c b\right)+\mathrm{BR}\left(H^{ \pm} \rightarrow c s\right)=1$. Right panel: in two-jet channel with one $b$-tag with $\mathrm{BR}\left(H^{ \pm} \rightarrow \tau \nu\right)=0.5$ and $\mathrm{BR}\left(H^{ \pm} \rightarrow c b\right)+\mathrm{BR}\left(H^{ \pm} \rightarrow c s\right)=0.5$.

results in the four-jet channel with two $b$-tags, with $\mathrm{BR}\left(H^{ \pm} \rightarrow c b\right)+\mathrm{BR}\left(H^{ \pm} \rightarrow c s\right)=1$. It can be seen that $\mathrm{BR}\left(H^{ \pm} \rightarrow c b\right)>0.4$ is required in order to obtain $S / \sqrt{B}>$ 2 for $M_{H^{ \pm}}=80 \mathrm{GeV}$ at a single experiment. In the right panel, we show the results for the two-jet channel with one $b$-tag, taking $\operatorname{BR}\left(H^{ \pm} \rightarrow \tau \nu\right)=0.5$, and $\operatorname{BR}\left(H^{ \pm} \rightarrow c b\right)+$ $\mathrm{BR}\left(H^{ \pm} \rightarrow c s\right)=0.5$. It can be seen that $\mathrm{BR}\left(H^{ \pm} \rightarrow c b\right)>$ 0.15 is required in order to obtain $S / \sqrt{B}>2$.
It is clear from the above plots that the four-jet channel with two $b$-tags offers the largest values of $S / \sqrt{B}$. In Table IV, the individual values of $S$ and $B$ (and $S / \sqrt{B}$ ) are shown for $M_{H^{ \pm}}=80,85$, and $89 \mathrm{GeV}$ in four-jet channels, with $\operatorname{BR}\left(H^{ \pm} \rightarrow c b\right)=0.8$ and $\operatorname{BR}\left(H^{ \pm} \rightarrow c s\right)=0.2$. In the $4 j 2 b$ channel, the signal does not satisfy $S \ll B$, which is a requirement for $S / \sqrt{B}$ to accurately represent the significance. If a more accurate formula for estimating

TABLE IV. Number of signal events $(S)$, number of background events $(B)$, and corresponding significances $\left(\frac{S}{\sqrt{B}}\right)$ in four-jet channels at single experiment at LEP2. Results are shown for $M_{H^{ \pm}}=80,85,89 \mathrm{GeV}$, with $\mathrm{BR}\left(H^{ \pm} \rightarrow c b\right)=0.8$ and $\mathrm{BR}\left(H^{ \pm} \rightarrow c s\right)=0.2$.

\begin{tabular}{|c|c|c|c|c|c|c|c|}
\hline & $80 \mathrm{GeV}$ & $85 \mathrm{GeV}$ & $89 \mathrm{GeV}$ & $80 \mathrm{GeV}$ & $85 \mathrm{GeV}$ & $89 \mathrm{GeV}$ & \\
\hline$M_{H^{ \pm}}$ & $S$ & $S$ & $S$ & $\frac{S}{\sqrt{B}}$ & $\frac{S}{\sqrt{B}}$ & $\frac{S}{\sqrt{B}}$ & $B$ \\
\hline $4 j 0 b$ & 69.50 & 46.01 & 29.07 & 2.08 & 1.38 & 0.87 & 1117.8 \\
\hline $4 j 1 b$ & 31.74 & 21.01 & 13.27 & 3.32 & 2.20 & 1.39 & 91.44 \\
\hline $4 j 2 b$ & 23.43 & 15.50 & 9.80 & 7.43 & 4.92 & 3.11 & 9.94 \\
\hline
\end{tabular}


TABLE V. Number of signal events $(S)$, number of background events $(B)$, and corresponding significances $\left(\frac{S}{\sqrt{B}}\right)$ in two-jet channels at a single experiment at LEP2. Results are shown for $M_{H^{ \pm}}=80,85,89 \mathrm{GeV}$, with $\mathrm{BR}\left(H^{ \pm} \rightarrow c b\right)=0.4, \mathrm{BR}\left(H^{ \pm} \rightarrow c s\right)=0.1$, and $\mathrm{BR}\left(H^{ \pm} \rightarrow \tau \nu\right)=0.5$.

\begin{tabular}{lccccccc}
\hline \hline & $80 \mathrm{GeV}$ & $85 \mathrm{GeV}$ & $89 \mathrm{GeV}$ & $80 \mathrm{GeV}$ & \multicolumn{2}{c}{$85 \mathrm{GeV}$} & \multicolumn{2}{c}{$89 \mathrm{GeV}$} \\
\cline { 2 - 6 }$M_{H^{ \pm}}$ & $S$ & $S$ & $S$ & $\frac{S}{\sqrt{B}}$ & $\frac{S}{\sqrt{B}}$ & $\frac{S}{\sqrt{B}}$ & \multicolumn{1}{c}{$B$} \\
\hline $2 j 0 b$ & 26.89 & 17.80 & 11.24 & 1.51 & 1.00 & 0.63 \\
$2 j 1 b$ & 15.28 & 10.11 & 6.39 & 4.08 & 2.70 & 1.71 & 14.04 \\
\hline \hline
\end{tabular}

the significance [50] is used for the $4 j 2 b$ channel, then the significance will be reduced compared to that given in Table IV.

From the numbers in Table IV, it can be seen that the background decreases significantly as each $b$-tag is applied, and there are still a significant number of events $(S \approx 9)$ in the four-jet channel with two $b$-tags for $M_{H^{ \pm}}=89 \mathrm{GeV}$. Around seven of nine background events in the $4 j 2 b$ channel are from the two-fermion background, and an invariant mass cut could further reduce this background (see later).

In Table $\mathrm{V}$, the individual values of $S$ and $B$ (and $S / \sqrt{B}$ ) are shown for $M_{H^{ \pm}}=80,85$, and $89 \mathrm{GeV}$ in two-jet channels, with $\mathrm{BR}\left(H^{ \pm} \rightarrow c b\right)=0.4, \mathrm{BR}\left(H^{ \pm} \rightarrow c s\right)=0.1$, and $\operatorname{BR}\left(H^{ \pm} \rightarrow \tau \nu\right)=0.5$. In the $2 j 1 b$ channel, the signal does not satisfy $S \ll B$, and if a more accurate formula for estimating the significance [50] is used, then the significance will be reduced compared to that given in Table V. Again, the background has decreased significantly with the $b$-tag, and there are still a reasonable number of events $(S \approx 6)$ in the two-jet channel with a $b$-tag for $M_{H^{ \pm}}=89 \mathrm{GeV}$.

As mentioned above in the discussion of Table IV, the two-fermion background accounts for most of the background in the $4 j 2 b$ channel. The invariant mass $\left(m_{j j}\right)$ of two of the four jets from the two-fermion background has a flat distribution (as can be seen in Fig. 1(h) of the OPAL

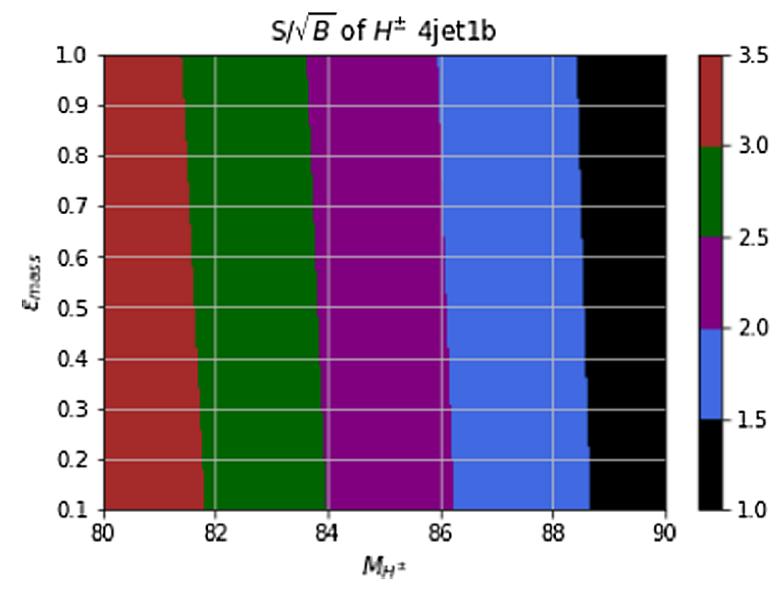

search in [39]), while the signal is mainly contained in the region of $m_{j j}$ between 80 and $89 \mathrm{GeV}$. Hence, we suggest that an invariant mass cut which only keeps jets satisfying $80 \mathrm{GeV}<m_{j j}<89 \mathrm{GeV}$ could further improve $S / \sqrt{B}$ in the $4 j 2 b$ channel. From Fig. 1(h) in [39], we estimate that such a cut could reduce the two-fermion background by a factor of 2, while preserving the majority of the signal events of an $H^{ \pm}$with a mass between 80 and $89 \mathrm{GeV}$. In Fig. 11, the effect of the invariant mass cut efficiency $\left(\epsilon_{\text {mass }}\right)$ on $S / \sqrt{B}$ in the four-jet channel with one and two $b$-tags is shown. For illustration, we vary $\epsilon_{\text {mass }}$ from 1 (i.e., no cut) to 0.1 , with values of $0.4<\epsilon_{\text {mass }}<0.5$ being suggested by Fig. 1(h) in [39]. This efficiency multiplies the two-fermion background only, and for simplicity we assume that the signal is not affected by the invariant mass cut (in reality some signal events would be lost due to the reconstructed peak at $M_{H^{ \pm}}$having a width). Taking $\epsilon_{\text {mass }}=0.4$ and $M_{H^{ \pm}}=80 \mathrm{GeV}$, one can see from the right panel (for the two $b$-tag channel) that $S / \sqrt{B}$ improves from around 7 $\left(\epsilon_{\text {mass }}=1\right)$ to $9\left(\epsilon_{\text {mass }}=0.4\right)$.

Finally, we comment on a slight excess of events of greater than $2 \sigma$ significance that is present in the LEP working group combination of the searches for $e^{+} e^{-} \rightarrow$ $H^{+} H^{-}$at all four experiments [43]. The excess occurs around $M_{H^{ \pm}}=89 \mathrm{GeV}, \mathrm{BR}\left(H^{ \pm} \rightarrow\right.$ hadrons $)=65 \%$ and $\operatorname{BR}\left(H^{ \pm} \rightarrow \tau \nu\right)=35 \%$, and in our earlier work [13,21] we

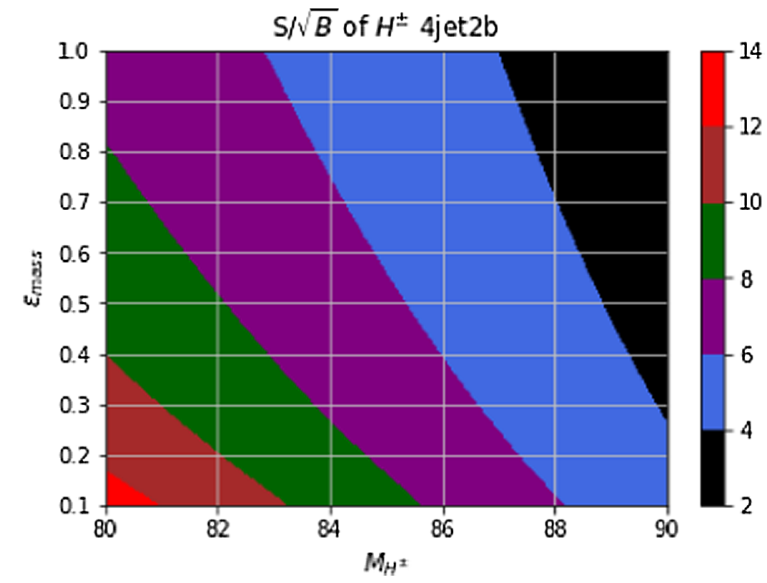

FIG. 11. Dependence of $S / \sqrt{B}$ on $M_{H^{ \pm}}$and on invariant mass cut ( $\left.\epsilon_{\text {mass }}\right)$ at a single LEP experiment, with $\mathrm{BR}\left(H^{ \pm} \rightarrow c b\right)=0.8$ and $\operatorname{BR}\left(H^{ \pm} \rightarrow c s\right)=0.2$. Left panel: four-jet channel with one $b$-tag. Right panel: four-jet channel with two $b$-tags. 
TABLE VI. Number of signal events $(S)$, number of background events $(B)$, and corresponding significances $\left(\frac{S}{\sqrt{B}}\right)$ in five channels at a single experiment at LEP2. Results are shown for $M_{H^{ \pm}}=88,89,90 \mathrm{GeV}$, with $\mathrm{BR}\left(H^{ \pm} \rightarrow c b\right)=0.5, \mathrm{BR}\left(H^{ \pm} \rightarrow c s\right)=0.15$, and $\operatorname{BR}\left(H^{ \pm} \rightarrow \tau \nu\right)=0.35$

\begin{tabular}{|c|c|c|c|c|c|c|c|}
\hline & $88 \mathrm{GeV}$ & $89 \mathrm{GeV}$ & $90 \mathrm{GeV}$ & $88 \mathrm{GeV}$ & $89 \mathrm{GeV}$ & $90 \mathrm{GeV}$ & \\
\hline$M_{H^{ \pm}}$ & $S$ & $S$ & $S$ & $\frac{S}{\sqrt{B}}$ & $\frac{S}{\sqrt{B}}$ & $\frac{S}{\sqrt{B}}$ & $B$ \\
\hline $4 j 0 b$ & 13.98 & 12.28 & 10.64 & 0.42 & 0.37 & 0.32 & 1117.8 \\
\hline $4 j 1 b$ & 6.47 & 5.68 & 4.93 & 0.68 & 0.59 & 0.52 & 91.44 \\
\hline $4 j 2 b$ & 4.43 & 3.89 & 3.37 & 1.41 & 1.23 & 1.07 & 9.94 \\
\hline $2 j 0 b$ & 11.65 & 10.23 & 8.87 & 0.65 & 0.57 & 0.5 & 316.9 \\
\hline $2 j 1 b$ & 6.43 & 5.65 & 4.89 & 1.72 & 1.51 & 1.31 & 14.04 \\
\hline
\end{tabular}

suggested the possibility of this being due to an $H^{ \pm}$of a 3HDM. If such an excess is genuine, and if a large fraction of the hadronic $\mathrm{BR}$ is from $H^{ \pm} \rightarrow c b$ decays, then $b$-tagging would increase the significance. In Table VI, we show the values of $S, B$, and $S / \sqrt{B}$ for $M_{H^{ \pm}}=88,89$, and $90 \mathrm{GeV}$. We take $\operatorname{BR}\left(H^{ \pm} \rightarrow c b\right)=50 \%$ and $\operatorname{BR}\left(H^{ \pm} \rightarrow c s\right)=15 \%$ (in order to obtain $\operatorname{BR}\left(H^{ \pm} \rightarrow\right.$ hadrons $)=65 \%$ ) and fix $\operatorname{BR}\left(H^{ \pm} \rightarrow \tau \nu\right)=35 \%$.

In Table VI, one can see that $S<B$ for all numbers given, and the $4 j 0 b$ and $2 j 0 b$ channels (i.e., the current searches) give significances of 0.37 and 0.57 , respectively, for $M_{H^{ \pm}}=89 \mathrm{GeV}$. These numbers are for a single LEP2 experiment, and so it is conceivable that the combination of four experiments could give the observed $2 \sigma$ excess, especially if there has been an upward fluctuation. In the $4 j 2 b$ and $2 j 1 b$ channels, these significances increase to 1.17 and 1.51, respectively, (i.e., a factor of 3 improvement), with the number of signal events $(S)$ still being above three events in each channel. Consequently, if the excess is genuine, then its significance could be significantly increased in the $4 j 2 b$ and $2 j 1 b$ channels, assuming that $\operatorname{BR}\left(H^{ \pm} \rightarrow c b\right)$ is large. As discussed in [21], such a signal might also show up at the LHC in the channel $t \rightarrow H^{ \pm} b$, which currently has sensitivity to the region $80 \mathrm{GeV}<M_{H^{ \pm}}<90 \mathrm{GeV}$ for $H^{ \pm} \rightarrow \tau \nu$ decays (but does not yet have sensitivity to $H^{ \pm} \rightarrow$ hadrons decays in this mass region). However, if the couplings $|X|$ and $|Y|$ [which determine $\left.\operatorname{BR}\left(t \rightarrow H^{ \pm} b\right)\right]$ are sufficiently small, then such an $H^{ \pm}$would remain hidden from LHC searches. At $e^{+} e^{-}$ colliders, the production channel $e^{+} e^{-} \rightarrow H^{+} H^{-}$does not depend on $|X|$ and $|Y|$, and a high luminosity $e^{+} e^{-}$collider would be able to probe the region $80 \mathrm{GeV}<M_{H^{ \pm}}<$ $90 \mathrm{GeV}$ irrespective of $|X|$ and $|Y|$.

\section{B. Prospects for detecting $H^{ \pm} \rightarrow c b$ at CEPC/FCC-ee}

Future $e^{+} e^{-}$colliders [51] are being discussed, which would offer precise measurements of the properties of the $125 \mathrm{GeV}$ neutral Higgs boson. Such colliders would also permit detailed studies of a light charged Higgs boson. There are two proposals for a circular $e^{+} e^{-}$collider with a period of operation at $\sqrt{s}=240 \mathrm{GeV}$ : CEPC in China [52] and FCC-ee [53] at CERN. These colliders would produce a large number of $\mathrm{H}^{+} \mathrm{H}^{-}$events with a mass of up to $M_{H^{ \pm}}=120 \mathrm{GeV}$. The integrated luminosity at this energy is expected to be of the order of $5 \mathrm{ab}^{-1}$, which is roughly several thousand times larger than the total integrated luminosity taken at a single LEP2 experiment $\left(0.6 \mathrm{fb}^{-1}\right)$. Two linear $e^{+} e^{-}$colliders are also being discussed, the International Linear Collider [54] and the Compact Linear Collider [55], which will both offer the possibility of energies much higher than $\sqrt{s}=240 \mathrm{GeV}$. In this work, we will consider the detection prospects of the decay channel $H^{ \pm} \rightarrow c b$ at $\sqrt{s}=240 \mathrm{GeV}$ only. As mentioned earlier, $\operatorname{BR}\left(H^{ \pm} \rightarrow c b\right)$ is expected to be at most of the order of $1 \%$ in the Type I, Type II, and leptonic specific 3HDMs. Only the flipped and democratic 3HDMs can have $\operatorname{BR}\left(H^{ \pm} \rightarrow c b\right)$ significantly larger than $1 \%$. Consequently, precise measurements of $\operatorname{BR}\left(H^{ \pm} \rightarrow c b\right)$ could shed light on which 3HDM Yukawa structure is realized. It is our aim to see if CEPC/FCC-ee would have sensitivity to smaller values (of the order of a few percent) for $\operatorname{BR}\left(H^{ \pm} \rightarrow c b\right)$. For the number of background events, we use the values from LEP2 (for which $\sqrt{s} \approx 200 \mathrm{GeV}$ ) for simplicity and scale them by the ratio of integrated luminosities $(1000 / 0.6 \approx 1670)$. The parameter $\epsilon_{c}$ was taken to be 0.06 in our analysis at LEP2. At CEPC/FCC-ee, we expect that this efficiency would be improved, and thus we vary it in the range $0.01<\epsilon_{c}<0.06$. A sizeable reduction in $\epsilon_{c}$ over its value at LEP2 would decrease the background coming from $c$ quarks faking $b$ quarks, thus significantly enhancing the significance of the signal. We keep $\epsilon_{b}$ and $\epsilon_{j}$ at the LEP2 values, because improvements in these parameters would not greatly increase the significance of the signal. The input parameters for the study of the detection prospects of $H^{ \pm} \rightarrow c b$ at $\sqrt{s}=$ $240 \mathrm{GeV}$ are summarized in Table III.

In Fig. 12, the dependence of $S / \sqrt{B}$ on $M_{H^{ \pm}}$and $\epsilon_{c}$ at $\sqrt{s}=240 \mathrm{GeV}$ is shown, with $\operatorname{BR}\left(H^{ \pm} \rightarrow c b\right)=0.05$ (which would only be possible in flipped/democratic 3HDMs) and $\operatorname{BR}\left(H^{ \pm} \rightarrow c s\right)=0.95$ (i.e., the decays to leptons are absent). The left panel is for the four-jet channel with one $b$-tag and the right panel is for the four-jet channel 

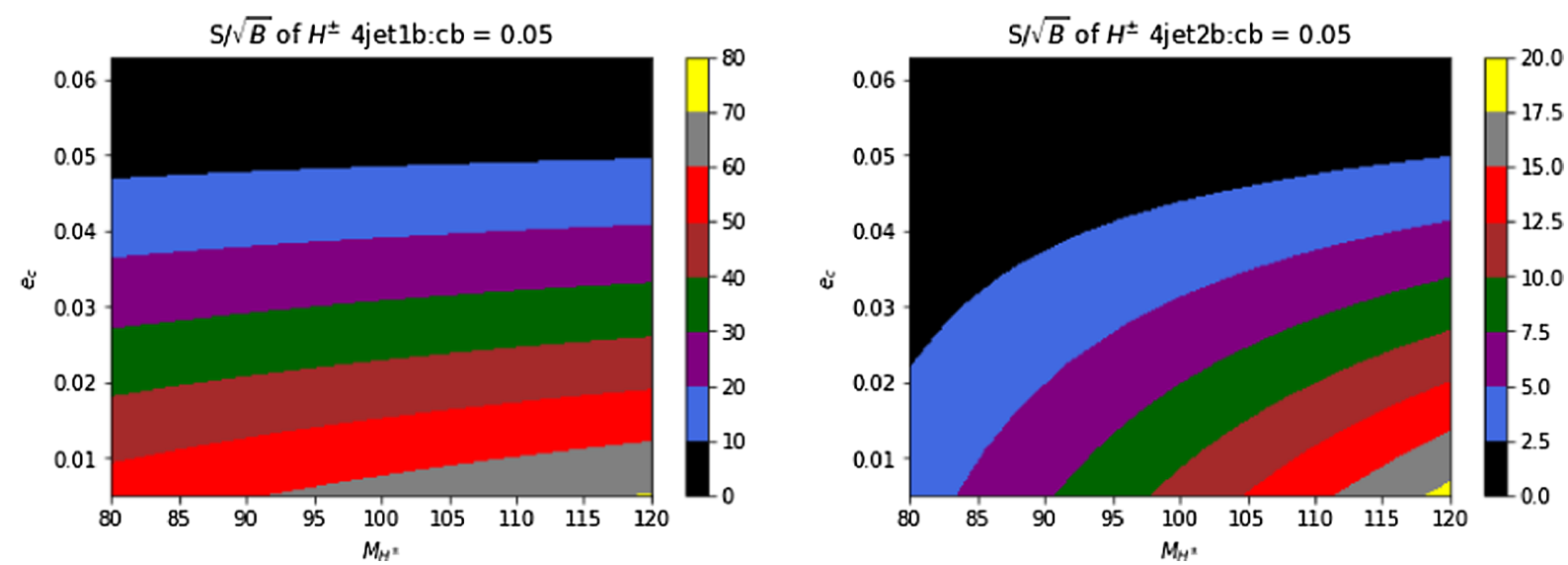

FIG. 12. Dependence of $S / \sqrt{B}$ on $M_{H^{ \pm}}$and $\epsilon_{c}$ at $\sqrt{s}=240 \mathrm{GeV}\left(\mathrm{CEPC} / \mathrm{FCC}\right.$-ee with $\left.1 \mathrm{ab}^{-1}\right)$, with $\mathrm{BR}\left(H^{ \pm} \rightarrow c b\right)=0.05$ and $\mathrm{BR}\left(H^{ \pm} \rightarrow c s\right)=0.95$. Left panel: four-jet channel with one $b$-tag. Right panel: four-jet channel with two $b$-tags.

with two $b$-tags. In the four-jet channel with one $b$-tag, it can be seen that very large values of $S / \sqrt{B}$ can be achieved (e.g., $S / \sqrt{B} \approx 30$ for $\epsilon_{c}=0.03$ and $M_{H^{ \pm}}=90 \mathrm{GeV}$ ), and thus precise measurements of $\mathrm{BR}\left(H^{ \pm} \rightarrow c b\right)$ would be obtained over a wide region of the plane $\left[M_{H^{ \pm}}, \epsilon_{c}\right]$. For

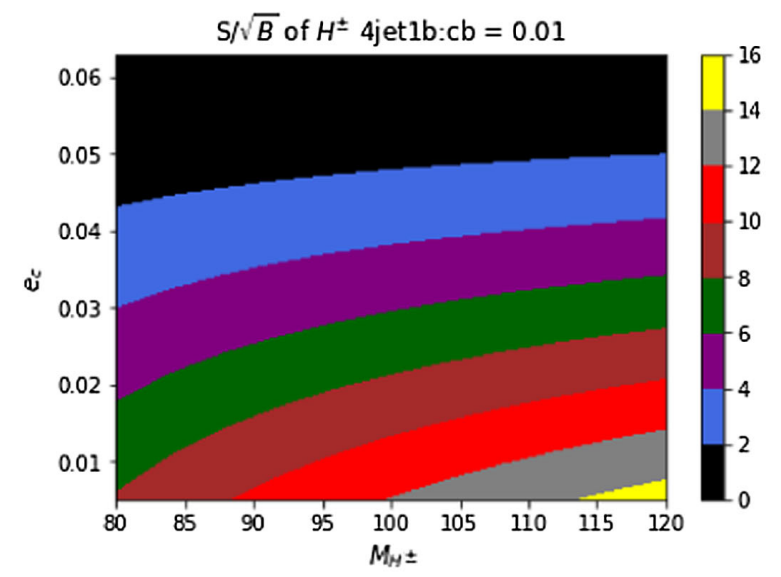

$\mathrm{BR}\left(H^{ \pm} \rightarrow c b\right)>0.05$, the values of $S / \sqrt{B}$ would be even larger. Note that the values of $S / \sqrt{B}$ are much lower in the four-jet channel with two $b$-tags (right panel). This is because $\operatorname{BR}\left(H^{ \pm} \rightarrow c b\right)=0.05$, leading to a reduced number of signal events with two $b$ quarks compared to the case

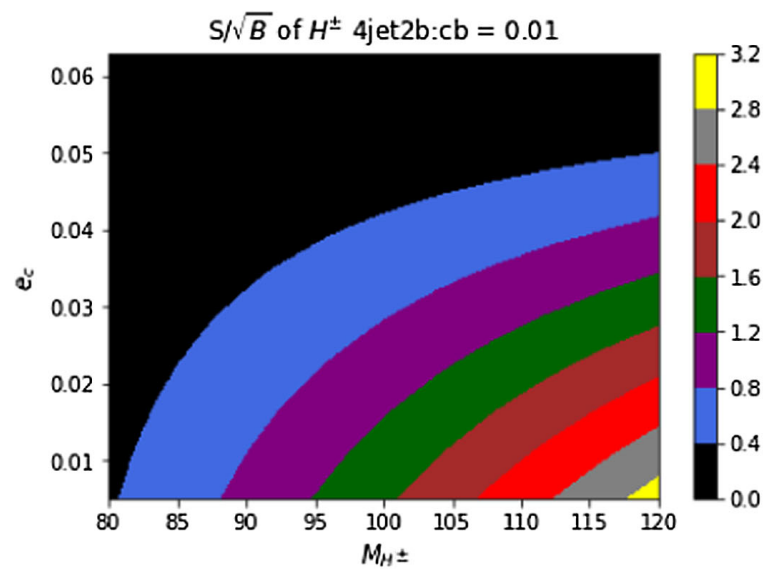

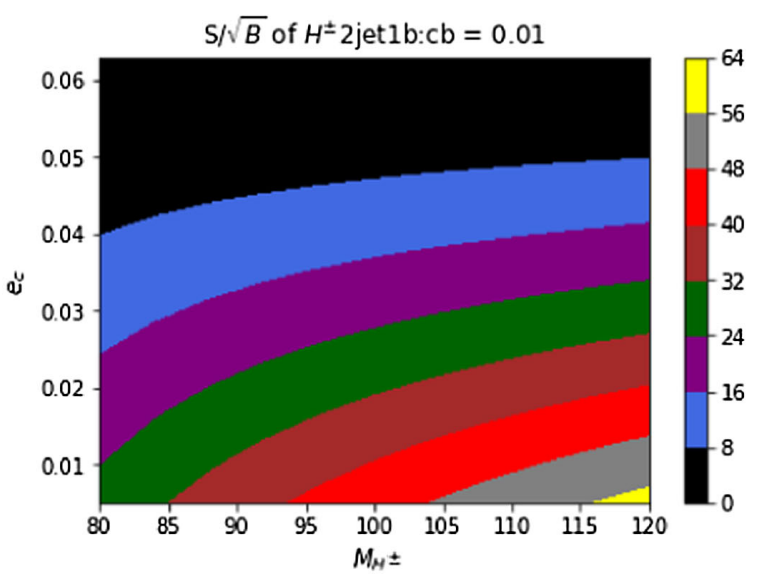

FIG. 13. Dependence of $S / \sqrt{B}$ on $M_{H^{ \pm}}$and $\epsilon_{c}$ at $\sqrt{s}=240 \mathrm{GeV}\left(\mathrm{CEPC} /\right.$ FCC-ee with $\left.1 \mathrm{ab}^{-1}\right)$, with $\mathrm{BR}\left(H^{ \pm} \rightarrow c b\right)=0.01$, $\mathrm{BR}\left(H^{ \pm} \rightarrow c s\right)=0.50$, and $\mathrm{BR}\left(H^{ \pm} \rightarrow \tau \nu\right)=0.49$. Top left panel: four-jet channel with one $b$-tag. Top right panel: four-jet channel with two $b$-tags. Bottom panel: two-jet channel with one $b$-tag. 
at LEP2 where the optimum scenario of $\mathrm{BR}\left(H^{ \pm} \rightarrow c b\right)=$ 0.8 was considered.

In Fig. 13, the dependence of $S / \sqrt{B}$ on $M_{H^{ \pm}}$and $\epsilon_{c}$ at $\sqrt{s}=240 \mathrm{GeV}$ is shown, with $\mathrm{BR}\left(H^{ \pm} \rightarrow c b\right)=0.01$, $\operatorname{BR}\left(H^{ \pm} \rightarrow c s\right)=0.50$, and $\operatorname{BR}\left(H^{ \pm} \rightarrow \tau \nu\right)=0.49$. The top left panel is for the four-jet channel with one $b$-tag, the top right panel is for the four-jet channel with two $b$ tags, and the bottom panel is for the two-jet channel with one $b$-tag. In the four-jet channel with one $b$-tag and the two-jet channel with one $b$-tag, a clear signal (and hence a precise measurement) can be achieved over a wide region of the plane $\left[M_{H^{ \pm}}, \epsilon_{c}\right]$. This would establish the presence of $H^{ \pm} \rightarrow c b$ decays even for $\operatorname{BR}\left(H^{ \pm} \rightarrow c b\right)=0.01$, a BR that is theoretically possible in all five Yukawa structures.

\section{CONCLUSIONS}

The decay channel $H^{ \pm} \rightarrow c b$ can have a large BR (up to $80 \%)$ in the flipped and democratic 3HDMs for $M_{H^{ \pm}}<M_{t}$ and be compatible with constraints from $b \rightarrow s \gamma$. The current search at the LHC (with $\sqrt{s}=8 \mathrm{TeV}, \mathcal{L}=20 \mathrm{fb}^{-1}$ ) for $t \rightarrow H^{ \pm} b$ followed by $H^{ \pm} \rightarrow c b$ is not sensitive to the region $80 \mathrm{GeV} \leq M_{H^{ \pm}} \leq 90 \mathrm{GeV}$, although sensitivity might be reached in future searches. LEP2 searched for $e^{+} e^{-} \rightarrow H^{+} H^{-}$, assuming the main decay channels to be $H^{ \pm} \rightarrow$ hadrons and $H^{ \pm} \rightarrow \tau \nu$. In the region $80 \mathrm{GeV} \leq M_{H^{ \pm}} \leq$ $90 \mathrm{GeV}$, a sizeable part of the plane $\left[\mathrm{BR}\left(H^{ \pm} \rightarrow\right.\right.$ hadrons $)$, $\left.M_{H^{ \pm}}\right]$is not excluded at LEP2 if $\operatorname{BR}\left(H^{ \pm} \rightarrow\right.$ hadrons $)$ is dominant. If $\operatorname{BR}\left(H^{ \pm} \rightarrow c b\right)$ were large, then more of the region $80 \mathrm{GeV} \leq M_{H^{ \pm}} \leq 90 \mathrm{GeV}$ could be probed at LEP2 by adding one or more $b$-tags to the existing search strategy. We evaluated the significances $(S / \sqrt{B})$ for $H^{ \pm} \rightarrow c b$ decays in three channels by taking the selection efficiencies and backgrounds from the OPAL searches and applying realistic $b$-tagging and fake $b$-tagging efficiencies. In the optimum scenario of $\mathrm{BR}\left(H^{ \pm} \rightarrow c b\right)=80 \%\left(\mathrm{BR}\left(H^{ \pm} \rightarrow c b\right)=40 \%\right.$ for 2-jets), it was shown that $S / \sqrt{B}$ as large as seven, three, and four could be obtained for $M_{H^{ \pm}}=80 \mathrm{GeV}$ in the three channels: (i) four-jet plus two $b$-tags, (ii) four-jet plus one $b$ tag, and (iii) two-jets plus one $b$-tag, respectively. These significances decrease to roughly $3,1.4$, and 1.7 , respectively, for $M_{H^{ \pm}}=89 \mathrm{GeV}$, but would be increased by combining all four experiments. Consequently, LEP 2 has the capability to exclude or discover a $H^{ \pm}$with a large $\mathrm{BR}\left(H^{ \pm} \rightarrow c b\right)$ and with a mass in the region $80 \mathrm{GeV} \leq$ $M_{H^{ \pm}} \leq 90 \mathrm{GeV}$. We commented on a $>2 \sigma$ excess at around $M_{H^{ \pm}}=89 \mathrm{GeV}$ and $\mathrm{BR}\left(H^{ \pm} \rightarrow\right.$ hadrons $) \approx 65 \%$ in the LEP working group combination. Under the assumption that such an excess is genuine and has a large $\operatorname{BR}\left(H^{ \pm} \rightarrow c b\right)$, it was shown that its significance could be increased significantly in two of the three channels with $b$-tagging. We encourage an updated LEP2 search for $H^{ \pm}$that includes $b$ tagging as suggested above. This would become especially important if the LHC eventually obtains evidence for an $H^{ \pm}$ with $80 \mathrm{GeV} \leq M_{H^{ \pm}} \leq 90 \mathrm{GeV}$ and a large $\operatorname{BR}\left(H^{ \pm} \rightarrow c b\right)$.

In contrast to hadron colliders, the cross section for $H^{ \pm}$ at LEP2 does not depend on the magnitude of the Yukawa couplings. Hence, a light $H^{ \pm}$with small Yukawa couplings could escape detection at the LHC, but be discovered at LEP2 or at future $e^{+} e^{-}$colliders. Even if a light $H^{ \pm}$is discovered at the LHC, future $e^{+} e^{-}$colliders would be able to measure its BRs much more precisely in order to shed light on the underlying Higgs structure. We evaluated $S / \sqrt{B}$ for $H^{ \pm} \rightarrow c b$ decays at a proposed $e^{+} e^{-}$collider (CEPC/FCC-ee) of $\sqrt{s}=240 \mathrm{GeV}$ and found that $\mathrm{BR}\left(H^{ \pm} \rightarrow c b\right)=1 \%$ (which is possible in all 2HDMs/ 3HDMs) would give a clear signal. In the context of 3HDMs, the flipped and democratic structures are the only ones which can have $\operatorname{BR}\left(H^{ \pm} \rightarrow c b\right)$ significantly greater than $1 \%$, and so precise measurements of this channel could provide evidence for these models.

\section{ACKNOWLEDGMENTS}

S. M. is supported in part through the NExT Institute and the STFC Consolidated Grant No. ST/L000296/1. S. M. and M.S. acknowledge the H2020-MSCA-RISE-2014 Grant No. 645722 (NonMinimalHiggs). M. S. thanks Professor Shinya Kanemura and Osaka University as well as Professor Tetsuo Shindou and Kogakuin University for hospitality where parts of this work were carried out.
[1] G. Aad et al. (ATLAS Collaboration), Phys. Lett. B 716, 1 (2012).

[2] S. Chatrchyan et al. (CMS Collaboration), Phys. Lett. B 716, 30 (2012).

[3] A. G. Akeroyd et al., Eur. Phys. J. C 77, 276 (2017).

[4] J. F. Gunion, H. E. Haber, G. L. Kane, and S. Dawson, Front. Phys. 80, 1 (2000).

[5] G. C. Branco, P. M. Ferreira, L. Lavoura, M. N. Rebelo, M. Sher, and J. P. Silva, Phys. Rep. 516, 1 (2012).
[6] S. L. Glashow and S. Weinberg, Phys. Rev. D 15, 1958 (1977); E. A. Paschos, Phys. Rev. D 15, 1966 (1977).

[7] I. P. Ivanov and E. Vdovin, Eur. Phys. J. C 73, 2309 (2013).

[8] V. Keus, S. F. King, and S. Moretti, J. High Energy Phys. 01 (2014) 052.

[9] Y. Grossman, Nucl. Phys. B426, 355 (1994).

[10] A. G. Akeroyd and W. J. Stirling, Nucl. Phys. B447, 3 (1995).

[11] G. Cree and H. E. Logan, Phys. Rev. D 84, 055021 (2011). 
[12] A. G. Akeroyd, S. Moretti, and J. Hernandez-Sanchez, Phys. Rev. D 85, 115002 (2012).

[13] A. G. Akeroyd, S. Moretti, K. Yagyu, and E. Yildirim, Int. J. Mod. Phys. A 32, 1750145 (2017).

[14] A. G. Akeroyd, Nucl. Phys. B544, 557 (1999).

[15] M. Misiak and M. Steinhauser, Eur. Phys. J. C 77, 201 (2017).

[16] T. Hermann, M. Misiak, and M. Steinhauser, J. High Energy Phys. 11 (2012) 036.

[17] M. Misiak et al., Phys. Rev. Lett. 114, 221801 (2015).

[18] A. M. Sirunyan et al. (CMS Collaboration), J. High Energy Phys. 11 (2018) 115.

[19] G. Aad et al. (ATLAS Collaboration), Eur. Phys. J. C 73, 2465 (2013).

[20] V. Khachatryan et al. (CMS Collaboration), J. High Energy Phys. 12 (2015) 178.

[21] A. G. Akeroyd, S. Moretti, and M. Song, Phys. Rev. D 98, 115024 (2018).

[22] A. Sopczak, Int. J. Mod. Phys. A 09, 1747 (1994).

[23] O. Eberhardt, U. Nierste, and M. Wiebusch, J. High Energy Phys. 07 (2013) 118.

[24] I. P. Ivanov, J. High Energy Phys. 07 (2010) 020; I. P. Ivanov and E. Vdovin, Phys. Rev. D 86, 095030 (2012); V. Keus, S. F. King, and S. Moretti, J. High Energy Phys. 01 (2014) 052; M. Maniatis and O. Nachtmann, J. High Energy Phys. 02 (2015) 058; 10 (2015) 149(E); S. Moretti and K. Yagyu, Phys. Rev. D 91, 055022 (2015).

[25] M. P. Bento, H. E. Haber, J. C. Romo, and J. P. Silva, J. High Energy Phys. 11 (2017) 095; H. E. Haber, O. M. Ogreid, P. Osland, and M. N. Rebelo, J. High Energy Phys. 01 (2019) 042.

[26] M. Jung, A. Pich, and P. Tuzon, J. High Energy Phys. 11 (2010) 003.

[27] M. Trott and M. B. Wise, J. High Energy Phys. 11 (2010) 157.

[28] H. E. Logan and D. MacLennan, Phys. Rev. D 81, 075016 (2010).

[29] V. M. Abazov et al. (D0 Collaboration), Phys. Lett. B 682, 278 (2009).

[30] T. Aaltonen et al. (CDF Collaboration), Phys. Rev. Lett. 103, 101803 (2009).

[31] S. Chatrchyan et al. (CMS Collaboration), J. High Energy Phys. 07 (2012) 143.

[32] G. Aad et al. (ATLAS Collaboration), J. High Energy Phys. 03 (2013) 076.
[33] G. Aad et al. (ATLAS Collaboration), J. High Energy Phys. 06 (2012) 039.

[34] G. Aad et al. (ATLAS Collaboration), J. High Energy Phys. 03 (2015) 088.

[35] V. Khachatryan et al. (CMS Collaboration), J. High Energy Phys. 11 (2015) 018.

[36] M. Aaboud et al. (ATLAS Collaboration), J. High Energy Phys. 09 (2018) 139.

[37] A. M. Sirunyan et al. (CMS Collaboration), J. High Energy Phys. 07 (2019) 142.

[38] S. Komamiya, Phys. Rev. D 38, 2158 (1988).

[39] G. Abbiendi et al. (OPAL Collaboration), Eur. Phys. J. C 72, 2076 (2012).

[40] A. Heister et al. (ALEPH Collaboration), Phys. Lett. B 543, 1 (2002).

[41] P. Achard et al. (L3 Collaboration), Phys. Lett. B 575, 208 (2003).

[42] J. Abdallah et al. (DELPHI Collaboration), Eur. Phys. J. C 34, 399 (2004).

[43] G. Abbiendi et al. (ALEPH and DELPHI and L3 and OPAL and LEP Collaborations), Eur. Phys. J. C 73, 2463 (2013).

[44] J. Abdallah et al. (DELPHI Collaboration), Eur. Phys. J. C 32, 185 (2004).

[45] G. Abbiendi et al. (OPAL Collaboration), Phys. Lett. B 609, 212 (2005).

[46] M. Tanabashi et al. (Particle Data Group), Phys. Rev. D 98, 030001 (2018).

[47] S. Schael et al. (ALEPH and DELPHI and L3 and OPAL Collaborations and LEP Working Group for Higgs Boson Searches), Eur. Phys. J. C 47, 547 (2006).

[48] T. Hebbeker and S. Roth, arXiv:1905.01111.

[49] J. Kile and J. von Wimmersperg-Toeller, J. High Energy Phys. 10 (2018) 116.

[50] G. Cowan, K. Cranmer, E. Gross, and O. Vitells, Eur. Phys. J. C 71, 1554 (2011); 73, 2501(E) (2013).

[51] J. de Blas et al., arXiv:1905.03764.

[52] J. B. Guimares da Costa et al. (CEPC Study Group), arXiv:1811.10545.

[53] A. Abada et al. (FCC Collaboration), Eur. Phys. J. C 79, 474 (2019).

[54] P. Bambade et al., arXiv:1903.01629.

[55] J. de Blas et al., CERN Yellow Rep. Monogr. 3, 1 (2018). 